\title{
Modified Krasnoselski-Mann iterative method for hierarchical fixed point problem and split mixed equilibrium problem
}

Jong Kyu Kim" and Prashanta Majee ${ }^{2}$

\section{"Correspondence:}

jongkyuk@kyungnam.ac.kr

'Department of Mathematics

Education, Kyungnam University,

Changwon, Gyeongnam, 51767,

Korea

Full list of author information is

available at the end of the article

\begin{abstract}
In this paper, we introduce a modified Krasnoselski-Mann type iterative method for capturing a common solution of a split mixed equilibrium problem and a hierarchical fixed point problem of a finite collection of $k$-strictly pseudocontractive nonself-mappings. Many of the algorithms for solving the split mixed equilibrium problem involve a step size which depends on the norm of a bounded linear operator. Since the computation of the operator norm is very difficult, we formulate our iterative algorithm in such a way that the implementation of the proposed algorithm does not require any prior knowledge of operator norm. Weak convergence results are established under mild conditions. We also establish strong convergence results for a certain class of hierarchical fixed point and split equilibrium problem. Our results generalize some important results in the recent literature.
\end{abstract}

MSC: $90 \mathrm{C} 33 ; 47 \mathrm{H} 10 ; 49 J 40$

Keywords: Nonexpansive mapping; Averaged mapping; Split mixed equilibrium problem; Fixed point problem

\section{Introduction}

Let $H_{1}$ and $H_{2}$ be two real Hilbert spaces with inner product $\langle\cdot, \cdot\rangle$ and norm $\|\cdot\|$. Let $C$ and $D$ be two nonempty closed and convex subsets of $H_{1}$ and $H_{2}$, respectively. A nonselfmapping $T: C \mapsto H_{1}$ is said to be $k$-strictly pseudocontractive if there exists a constant $k \in[0,1)$ such that

$$
\|T x-T y\|^{2} \leq\|x-y\|^{2}+k\|(I-T) x-(I-T) y\|^{2}, \quad \forall x, y \in H_{1} .
$$

If $k=0$, then $T$ is a nonexpansive nonself-mapping.

For a mapping $T: C \mapsto H_{1}$, the fixed point problem is to find $x \in C$ such that $x=T x$. The set of all fixed points of $T$ is denoted by $\operatorname{Fix}(T)$.

Moudafi and Mainge [24] considered the following hierarchical fixed point problem (in short, HFPP) for a nonexpansive self-mapping $T$ with respect to another nonexpansive

(c) The Author(s) 2020. This article is licensed under a Creative Commons Attribution 4.0 International License, which permits use, sharing, adaptation, distribution and reproduction in any medium or format, as long as you give appropriate credit to the original author(s) and the source, provide a link to the Creative Commons licence, and indicate if changes were made. The images or other third party material in this article are included in the article's Creative Commons licence, unless indicated otherwise in a credit line to the material. If material is not included in the article's Creative Commons licence and your intended use is not permitted by statutory regulation or exceeds the permitted use, you will need to obtain permission directly from the copyright holder. To view a copy of this licence, visit http://creativecommons.org/licenses/by/4.0/. 
self-mapping $S$ on $C$ in the following way: Find $x^{*} \in \operatorname{Fix}(T)$ such that

$$
\left\langle x^{*}-S x^{*}, x^{*}-x\right\rangle \leq 0, \quad \forall x \in \operatorname{Fix}(T) .
$$

Let us denote the solution set of the HFPP (1.1) as $\mathcal{S}=\left\{x^{*} \in \operatorname{Fix}(T):\left\langle x^{*}-S x^{*}, x^{*}-x\right\rangle \leq\right.$ $0, \forall x \in \operatorname{Fix}(T)\}$.

We can check that $x^{*}$ is a solution of the HFPP (1.1) if and only if $x^{*}=P_{\operatorname{Fix}(T)} \circ S x^{*}$, where $P_{\mathrm{Fix}(T)}$ is the metric projection of $H_{1}$ onto $\operatorname{Fix}(T)$.

By using the definition of the normal cone to $\operatorname{Fix}(T)$, i.e.,

$$
N_{\operatorname{Fix}(T)}= \begin{cases}\left\{u \in H_{1}:\langle y-x, u\rangle \leq 0, \forall y \in \operatorname{Fix}(T)\right\}, & \text { if } x \in \operatorname{Fix}(T), \\ \emptyset, & \text { otherwise }\end{cases}
$$

we can easily prove that HFPP (1.1) is equivalent to the variational inclusion

$$
0 \in(I-S) x^{*}+N_{\mathrm{Fix}(T)} x^{*}
$$

We note that based on the relation $x^{*}=P_{\operatorname{Fix}(T)} \circ S x^{*}, \operatorname{HFPP}(1.1)$ has an iterative algorithm $x_{n+1}=P_{\mathrm{Fix}(T)} \circ S x_{n}$. This iterative method converges if the mapping $P_{\mathrm{Fix}(T)} \circ S$ has a fixed point and $S$ is averaged not just nonexpansive. Disadvantage of this method is that the calculation of $P_{\mathrm{Fix}(T)} \circ S$ is usually not easy. To overcome this, Moudafi [22] introduced an algorithm which uses $T$ itself, rather than $P_{\mathrm{Fix}(T)} \circ S$. Moudafi introduced the iterative method in the following way: For starting point $x_{0} \in C$, define $\left\{x_{n}\right\}$ by

$$
x_{n+1}=\left(1-\alpha_{n}\right) x_{n}+\alpha_{n}\left(\sigma_{n} S x_{n}+\left(1-\sigma_{n}\right) T x_{n}\right) \text {, }
$$

where $\left\{\alpha_{n}\right\}$ and $\left\{\sigma_{n}\right\}$ are two real sequences in $(0,1)$.

Problems (1.1) are often used in the area of optimization and related fields, such as signal processing and image reconstruction (see $[4,6,11,14-20,25,27-30,32-36]$ and the references therein).

On the other hand, for a bifunction $F: C \times C \rightarrow \mathbb{R}$, an equilibrium problem is defined by

find $x \in C$ such that $F(x, y) \geq 0, \quad$ for all $y \in C$.

The solution set of this problem is denoted as $\operatorname{EP}(F, C)$. Equilibrium problem includes variational inequality problem, optimization problem, the Nash equilibrium problem, saddle point problem, complementarity problem, convex differential optimization etc. as special cases (see Blum and Oettli [1]).

In 2009, Marino et al. [21] introduced an iterative method to find common solutions of the following system of equilibrium problem and hierarchical fixed point problem:

$$
\left\{\begin{array}{l}
\text { find } x^{*} \in C \text { such that } F\left(x^{*}, y\right) \geq 0, \quad \forall y \in C ; \quad \text { and } \\
x^{*} \in \operatorname{Fix}(T) \text { such that }\left\langle x^{*}-f\left(x^{*}\right), x^{*}-y\right\rangle \leq 0, \quad \forall y \in \operatorname{Fix}(T),
\end{array}\right.
$$

where $F$ is a bifunction, $f$ is a $\rho$-contraction and $T$ is a nonexpansive self-mapping. 
In 2011, Moudafi [23] introduced and studied the following split equilibrium problem $\mathrm{S}_{p} \mathrm{EP}$ : Suppose $F_{1}: C \times C \rightarrow \mathbb{R}$ and $F_{2}: D \times D \rightarrow \mathbb{R}$ are two bifunctions and $A: H_{1} \rightarrow H_{2}$ is a bounded linear operator. Then the split equilibrium problem is to find $x^{*} \in C$ such that

$$
F_{1}\left(x^{*}, x\right) \geq 0, \quad \forall x \in C .
$$

and $y^{*}=A x^{*} \in D$ solves

$$
F_{2}\left(y^{*}, y\right) \geq 0, \quad \forall y \in D .
$$

In 2012, Censor et al. [7] introduced and studied the following split variational inequality problem $\mathrm{S}_{p}$ VIP: Find $x^{*} \in C$ such that

$$
\left\langle g_{1}\left(x^{*}\right), y-x^{*}\right\rangle \geq 0, \quad \forall x \in C,
$$

and $y^{*}=A x^{*} \in Q$ solves

$$
\left\langle g_{2}\left(x^{*}\right), y-x^{*}\right\rangle \geq 0, \quad \forall y \in D,
$$

where $g_{1}: C \rightarrow H_{1}$ and $g_{2}: D \rightarrow H_{2}$ are two nonlinear mappings, and $A: H_{1} \rightarrow H_{2}$ is a bounded linear operator.

Very recently, Kazmi et al. [13] have introduced and analyzed a Krasnoselski-Mann iteration method for finding a common solution of HFPP (1.1) and the following split mixed equilibrium problem $S_{p} M E P$ : Find $x^{*} \in C$ such that

$$
F_{1}\left(x^{*}, x\right)+\left\langle g_{1}\left(x^{*}\right), y-x^{*}\right\rangle \geq 0, \quad \forall x \in C,
$$

and $y^{*}=A x^{*} \in Q$ solves

$$
F_{2}\left(y^{*}, y\right)+\left\langle g_{2}\left(x^{*}\right), y-x^{*}\right\rangle \geq 0, \quad \forall y \in D
$$

The solution set of $\mathrm{S}_{p} \mathrm{MEP}$ is denoted by $\Gamma=\left\{p \in \operatorname{MEP}\left(F_{1}, g_{1}\right): A p \in \operatorname{MEP}\left(F_{2}, g_{2}\right)\right\}$. To solve the $\mathrm{S}_{p} \mathrm{MEP}$ (1.9)-(1.10) and HFPP (1.1), Kazmi et al. [13] introduced the following algorithm: For the starting point $x_{0} \in C$, define $\left\{x_{n}\right\}$ by

$$
\left\{\begin{array}{l}
u_{n}=\left(1-\alpha_{n}\right) x_{n}+\alpha_{n}\left(\sigma_{n} S x_{n}+(1-\sigma) T x_{n}\right), \\
x_{n+1}=U\left(u_{n}+\gamma A^{*}(V-I) A u_{n}\right), \quad n \geq 1,
\end{array}\right.
$$

where $S, T$ are nonexpansive self-mappings on $C$ and the step size $\gamma \in\left(0, \frac{1}{L}\right), L$ is the spectral radius of the operator $A^{*} A$ and $A^{*}$ is the adjoint of the bounded linear operator $A$.

Motivated by the above results, we revisit the problem considered by Kazmi et al. [13]. We introduce and analyze a modified Krasnoselski-Mann type iterative method with the help of averaged mappings for finding a common solution of the HFPP (1.1) of a finite collection of $k$-strictly pseudocontractive non-self-mappings and $\mathrm{S}_{p} \mathrm{MEP}$ (1.9)-(1.10). Our 
work can be seen as an extension of HFPP $(1.1)[13,22]$ from single nonexpansive selfmapping to a finite collection of $k$-strictly pseudocontractive nonself-mappings. The authors in $[10,13]$ have selected the step size $\gamma$ in the interval $\left(0, \frac{1}{L}\right)$, where $L$ is the spectral radius of the operator $A^{*} A$ and $A^{*}$ is the adjoint of the bounded linear operator $A$.

It is well known that the computation or an estimate of the spectral radius of a given operator is very difficult at times. This makes the implementation of the proposed algorithm very difficult. In our iterative method, we give an explicit formula to choose the step size, which does not require any prior knowledge of operator norm. We also establish strong convergence results for a certain class of hierarchical fixed point and split equilibrium problem.

We organize the paper in the following way. Some basic definitions and lemmas are given in Sect. 2. In Sect. 3, we present our modified iterative methods for a split mixed equilibrium problem and hierarchical fixed point problem. Finally, we make some remarks to highlight the main contribution of this paper.

\section{Preliminaries}

In order to prove our main results, we recall some basic definitions and lemmas, which will be needed in the sequel. Let $H_{1}$ be a real Hilbert space and $C$ be a nonempty closed convex subset of $H_{1}$. Let the symbols " $\rightarrow$ " and " $\rightarrow$ " denote the weak and strong convergence, respectively. We know that in a Hilbert space $H_{1}$, the following properties hold:

$$
\|x-y\|^{2} \leq\|x\|^{2}-\|y\|^{2}-2\langle x-y, y\rangle, \quad \forall x, y \in H_{1}
$$

and

$$
2\langle x, y\rangle=\|x\|^{2}+\|y\|^{2}-\|x-y\|^{2}=\|x+y\|^{2}-\|x\|^{2}-\|y\|^{2}, \quad \forall x, y \in H_{1} .
$$

It is well known that every nonexpansive mapping $T: H_{1} \rightarrow H_{1}$ satisfies the inequality:

$$
\langle(x-T x)-(y-T y), T y-T x\rangle \leq \frac{1}{2}\|(T x-x)-(T y-y)\|^{2}, \quad \forall(x, y) \in H_{1} \times H_{1} .
$$

Therefore for all $(x, y) \in H_{1} \times \operatorname{Fix}(T)$, we get

$$
\langle x-T x, y-T x\rangle \leq \frac{1}{2}\|(T x-x)\|^{2} .
$$

A mapping $T: H_{1} \rightarrow H_{1}$ is said to be monotone if

$$
\langle T x-T y, x-y\rangle \geq 0, \quad \forall x, y \in H_{1} .
$$

$T$ is said to be $\alpha$-inverse strongly monotone if there exists a $\alpha>0$ such that

$$
\langle T x-T y, x-y\rangle \geq \alpha\|T x-T y\|^{2}, \quad \forall x, y \in H_{1} .
$$

$T$ is said to be firmly nonexpansive if

$$
\langle T x-T y, x-y\rangle \geq\|T x-T y\|^{2}, \quad \forall x, y \in H_{1} .
$$


A mapping $P_{C}$ is said to be metric projection from $H_{1}$ onto $C$ if for every $x \in H_{1}$, there exists a unique nearest point in $C$ denoted by $P_{C}(x)$ such that

$$
\left\|x-P_{C}(x)\right\| \leq\|x-y\|, \quad \forall y \in C .
$$

It is well known that $P_{C}$ is nonexpansive and firmly nonexpansive. Moreover, $P_{C}$ is characterized by the following property:

$$
\left\langle x-P_{C}(x), y-P_{C}(x)\right| \leq 0, \quad \forall x \in H_{1}, y \in C .
$$

A multivalued mapping $M: H_{1} \rightarrow 2^{H_{1}}$ is called monotone if for any $x, y \in H_{1}$

$$
\langle u-v, x-y\rangle \geq 0, \quad \forall u \in M x, v \in M y .
$$

For a multivalued mapping $M, \operatorname{graph}(M)$ is defined by $\operatorname{graph}(M):=\left\{(x, u) \in H_{1} \times H_{1}: u \in\right.$ $M x\}$. A multivalued monotone mapping $M: H_{1} \rightarrow 2^{H_{1}}$ is said to be maximal monotone if $\operatorname{graph}(M)$ is not properly contained in the graph of any other monotone mapping. It is well known that a multivalued monotone mapping is maximal monotone if for $(x, u) \in H_{1} \times H_{1}$, $\langle x-y, u-v\rangle \geq 0$ for every $(y, v) \in \operatorname{graph}(M)$ implies that $u \in M x$. Let $M: H_{1} \rightarrow 2^{H_{1}}$ be a multivalued maximal monotone operator. Then the resolvent mapping $J_{\lambda}^{M}: H_{1} \rightarrow H_{1}$ associated with $M$ is defined by

$$
J_{\lambda}^{M}(x):=(I+\lambda M)^{-1}(x), \quad \forall x \in H_{1},
$$

for some $\lambda>0$, where $I$ is the identity operator on $H_{1}$. It is well known that for all $\lambda>0$ the resolvent operator is single-valued, nonexpansive and firmly nonexpansive. Also, we know that $\operatorname{Fix}\left(J_{\lambda}^{M}\right)=M^{-1}(0)$.

Definition 2.1 A mapping $T: H_{1} \rightarrow H_{1}$ is said to be an averaged mapping if there exists some number $\alpha \in(0,1)$ such that $T=(1-\alpha) I+\alpha S$, where $I: H_{1} \rightarrow H_{1}$ is the identity mapping and $S: H_{1} \rightarrow H_{1}$ is a nonexpansive mapping. An averaged mapping is also nonexpansive and $\operatorname{Fix}(S)=\operatorname{Fix}(T)$.

Lemma $2.2([3,4])$ If the mappings $\left\{T_{i}\right\}_{i=1}^{N}$ are averaged and have a common fixed point, then

$$
\bigcap_{i=1}^{N} \operatorname{Fix}\left(T_{i}\right)=\operatorname{Fix}\left(T_{1} T_{2} \cdots T_{N}\right) .
$$

In particular, for $N=2, \operatorname{Fix}\left(T_{1}\right) \cap \operatorname{Fix}\left(T_{2}\right)=\operatorname{Fix}\left(T_{1} T_{2}\right)=\operatorname{Fix}\left(T_{2} T_{1}\right)$.

Lemma 2.3 ([37]) Assume that $S: C \rightarrow H_{1}$ is a k-strictly pseudocontractive mapping. Define a mapping $T$ by $T x=\alpha x+(1-\alpha)$ Sx for all $x \in H_{1}$, where $\alpha \in[k, 1)$. Then $T$ is a nonexpansive mapping with $\operatorname{Fix}(T)=\operatorname{Fix}(S)$.

Lemma 2.4([37]) Let $T: C \rightarrow H_{1}$ be a $k$-strictly pseudocontractive mapping with $\operatorname{Fix}(T) \neq$ Ø. Then $\operatorname{Fix}\left(P_{C} T\right)=\operatorname{Fix}(T)$. 
Lemma 2.5 (Demiclosedness principle [12]) Let C be a nonempty closed convex subset of a real Hilbert space $H_{1}$ and let $T: C \rightarrow C$ be a nonexpansive mapping. If $\left\{x_{n}\right\}$ is a sequence in $C$ weakly converging to $x \in C$ and $\left\{(I-T) x_{n}\right\}$ converges strongly to $y \in C$, then $(I-T) x=y$. In particular, if $y=0$, then $x \in \operatorname{Fix}(T)$.

Assumption A ([1]) Let $F: C \times C \rightarrow \mathbb{R}$ be a bifunction which satisfies the following assumptions:

(i) $F(x, x) \geq 0, \forall x \in C$;

(ii) $F$ is monotone, i.e., $F(x, y)+F(y, x) \leq 0, \forall x, y \in C$;

(iii) $F$ is upper hemicontinuous, i.e., for each $x, y, z \in C$,

$$
\limsup _{t \rightarrow 0} F(t z+(1-t) x, y) \leq F(x, y)
$$

(iv) For each $x \in C$ fixed, the function $y \mapsto F(x, y)$ is convex and lower semicontinuous.

Lemma 2.6 ([9]) Assume that the bifunction $F: C \times C \rightarrow \mathbb{R}$ satisfies Assumption A. For $r>0$ and $x \in H_{1}$, define a mapping $T_{r}^{F}: H_{1} \rightarrow C$ as follows:

$$
T_{r}^{F}(x):=\left\{z \in C: F(z, y)+\frac{1}{r}\langle y-z, z-x\rangle \geq 0, \forall y \in C\right\} .
$$

Then the following properties hold:

(i) $T_{r}^{F}$ is nonempty and single-valued.

(ii) $T_{r}^{F}$ is firmly nonexpansive, i.e., $\left\|T_{r}^{F} x-T_{r}^{F} y\right\|^{2} \leq\left\langle T_{r}^{F} x-T_{r}^{F} y, x-y\right\rangle, \forall x, y \in H_{1}$.

(iii) $\operatorname{Fix}\left(T_{r}^{F}\right)=\operatorname{EP}(F, C)$.

(iv) $\mathrm{EP}(F, C)$ is closed and convex.

Furthermore, assume that $F_{2}: D \times D \rightarrow \mathbb{R}$ satisfy the conditions in Assumption A. For $s>0$ and for all $w \in H_{2}$, define a mapping $T_{s}^{F_{2}}: H_{2} \rightarrow D$ as follows:

$$
T_{s}^{F_{2}}(w):=\left\{d \in D: F(d, e)+\frac{1}{s}\langle e-d, d-w\rangle \geq 0, \forall e \in D\right\} .
$$

Then we easily observe that $T_{s}^{F_{2}}$ is nonempty, single-valued and firmly nonexpansive. Also, $\operatorname{EP}\left(F_{2}, D\right)$ is closed and convex, and $\operatorname{Fix}\left(T_{s}^{F_{2}}\right)=\operatorname{EP}\left(F_{2}, D\right)$, where $\operatorname{EP}\left(F_{2}, D\right)$ is the solution of the following equilibrium problem: Find $y^{*} \in D$ such that

$$
F_{2}\left(y^{*}, y\right) \geq 0, \quad \forall y \in D .
$$

Lemma 2.7 ([8]) Let $\left\{\delta_{n}\right\}$ and $\left\{\gamma_{n}\right\}$ be non-negative sequences satisfying $\sum_{n=0}^{\infty} \delta_{n}<+\infty$ and $\gamma_{n+1} \leq \gamma_{n}+\delta_{n}$ for all $n \in \mathbb{N}$. Then $\left\{\gamma_{n}\right\}$ is a convergent sequence.

Definition $2.8([2,24])$ A sequence $\left\{M_{n}\right\}$ of maximal monotone mappings defined on $H_{1}$ is said to be graph convergent to a multivalued mapping $M$ if $\left\{\operatorname{graph}\left(M_{n}\right)\right\}$ converges to 
$\operatorname{graph}(M)$ in the Kuratowski-Painlevé sense, that is,

$$
\limsup _{n \rightarrow \infty} \operatorname{graph}\left(M_{n}\right) \subset \operatorname{graph}(M) \subset \liminf _{n \rightarrow \infty} \operatorname{graph}\left(M_{n}\right)
$$

Lemma 2.9 ([9]) We have the following statements:

(i) Let $M$ be a maximal monotone mapping on $H_{1}$. Then $\left\{t_{n}{ }^{-1} M\right\}$ is graph convergent to $N_{M^{-1} 0}$ as $t_{n} \rightarrow 0$ provided that $M^{-1} 0 \neq \emptyset$.

(ii) Let $\left\{M_{n}\right\}$ be a sequence of maximal monotone mappings on $H_{1}$ which is graph convergent to a mapping $M$ defined on $H_{1}$. If $B$ is a Lipschitz maximal monotone mapping on $H_{1}$, then $\left\{B+M_{n}\right\}$ is graph convergent to $B+M$ and $B+M$ is maximal monotone.

\section{Main results}

In this section, we state and prove our main results of the paper. First we will study the weak convergence theorem.

Theorem 3.1 Let $H_{1}$ and $H_{2}$ be two Hilbert spaces. Let $C$ and $D$ be nonempty closed and convex subset of $H_{1}$ and $H_{2}$, respectively. Let $A: H_{1} \rightarrow H_{2}$ be a bounded linear operator. Suppose $F_{1}: C \times C \rightarrow \mathbb{R}$ and $F_{2}: D \times D \rightarrow \mathbb{R}$ are two bifunctions which satisfy Assumption $\mathrm{A}$ and $F_{2}$ is upper semicontinuous. Let $g_{1}: C \rightarrow H_{1}$ and $g_{2}: D \rightarrow H_{2}$ be $\eta_{1}$ - and $\eta_{2}$ inverse strongly monotone mappings, respectively. Let $S: C \rightarrow C$ be a nonexpansive selfmapping and $\left\{T_{i}\right\}_{i=1}^{N}: C \rightarrow H_{1}$ be $k_{i}$-strictly pseudocontractive nonself-mappings. Assume that

$$
\mathcal{F}=\Gamma \cap \mathcal{S} \neq \emptyset .
$$

Define a sequence $\left\{x_{n}\right\}$ as follows:

$$
\left\{\begin{array}{l}
x_{0} \in C, \\
u_{n}=\left(1-\alpha_{n}\right) x_{n}+\alpha_{n}\left(\tau_{n} S x_{n}+\left(1-\tau_{n}\right) T_{N}^{n} T_{N-1}^{n} \cdots T_{1}^{n} x_{n}\right), \\
x_{n+1}=U\left(u_{n}+\gamma_{n} A^{*}(V-I) A u_{n}\right), \quad n \geq 1,
\end{array}\right.
$$

where $U=T_{r_{n}}^{F_{1}}\left(I-r_{n} g_{1}\right), V=T_{r_{n}}^{F_{2}}\left(I-r_{n} g_{2}\right), T_{i}^{n}=\left(1-\delta_{n}^{i}\right) I+\delta_{n}^{i} P_{C}\left(\beta_{i} I+\left(1-\beta_{i}\right) T_{i}\right), 0 \leq k_{i} \leq$ $\beta_{i}<1, \delta_{n}^{i} \in(0,1)$ for $i=1,2, \ldots, N$ and

$$
\gamma_{n}=\frac{\sigma_{n}\left\|\left(T_{r_{n}}^{F_{2}}\left(I-r_{n} g_{2}\right)-I\right) A x_{n}\right\|}{\left\|A^{*}\left(T_{r_{n}}^{F_{2}}\left(I-r_{n} g_{2}\right)-I\right) A x_{n}\right\|}, \quad 0<a \leq \sigma_{n} \leq b<1 .
$$

Let $\left\{\alpha_{n}\right\},\left\{\tau_{n}\right\}$ be two real sequences in $(0,1)$ and $\left\{r_{n}\right\} \subset(0, \alpha)$, where $\alpha=2 \min \left\{\eta_{1}, \eta_{2}\right\}$. Suppose the following conditions are satisfied:

(i) $\sum_{n=0}^{\infty} \tau_{n}<\infty$;

(ii) $\lim _{n \rightarrow \infty} \frac{\left\|x_{n}-u_{n}\right\|}{\alpha_{n} \tau_{n}}=0$;

(iii) $\liminf _{n \rightarrow \infty} r_{n}>0$;

(iv) $\lim _{n \rightarrow \infty}\left|\delta_{n+1}^{i}-\delta_{n}^{i}\right|=0$ for $i=1,2, \ldots, N$.

Then the sequence $\left\{x_{n}\right\}$ converges weakly to $x^{*} \in \mathcal{F}$ 
Proof Since our method easily deduce the general case, we only prove the theorem for $N=2$. Since $g_{1}: H_{1} \rightarrow H_{1}$ is $\eta_{1}$-strongly monotone mapping, for any $x, y \in H_{1}$, we get

$$
\begin{aligned}
\left\|\left(I-r_{n} g_{1}\right) x-\left(I-r_{n} g_{1}\right) y\right\|^{2} & =\left\|(x-y)-r_{n}\left(g_{1} x-g_{1} y\right)\right\|^{2} \\
& \leq\|x-y\|^{2}-r_{n}\left(2 \eta_{1}-r_{n}\right)\left\|g_{1} x-g_{1} y\right\|^{2} \\
& \leq\|x-y\|^{2} .
\end{aligned}
$$

This means that $\left(I-r_{n} g_{1}\right)$ is nonexpansive. Similarly, we can show that $\left(I-r_{n} g_{2}\right)$ is a nonexpansive mapping. Thus, $U$ and $V$ are also nonexpansive mappings. Let $x^{*} \in \mathcal{F}$. From Lemma 2.2, Lemma 2.3 and Lemma 2.4, we get $x^{*}=T_{2}^{n} T_{1}^{n} x^{*}$. Hence, we have

$$
\begin{aligned}
\left\|u_{n}-x^{*}\right\|= & \left\|\left(1-\alpha_{n}\right) x_{n}+\alpha_{n}\left(\tau_{n} S x_{n}+\left(1-\tau_{n}\right) T_{2}^{n} T_{1}^{n} x_{n}\right)-x^{*}\right\| \\
\leq & \left(1-\alpha_{n}\right)\left\|x_{n}-x^{*}\right\|+\alpha_{n}\left[\tau_{n}\left\|S x_{n}-x^{*}\right\|+\left(1-\tau_{n}\right)\left\|T_{2}^{n} T_{1}^{n} x_{n}-x^{*}\right\|\right] \\
\leq & \left(1-\alpha_{n}\right)\left\|x_{n}-x^{*}\right\|+\alpha_{n}\left[\tau_{n}\left\|x_{n}-x^{*}\right\|+\left(1-\tau_{n}\right)\left\|x_{n}-x^{*}\right\|\right] \\
& +\alpha_{n} \tau_{n}\left\|S x^{*}-x^{*}\right\| \\
= & \left\|x_{n}-x^{*}\right\|+\alpha_{n} \tau_{n}\left\|S x^{*}-x^{*}\right\| .
\end{aligned}
$$

Also, since $x^{*} \in \mathcal{F}$, we have $U x^{*}=x^{*}$ and $V A x^{*}=A x^{*}$.

Let $v_{n}=u_{n}+\gamma A^{*}(V-I) A u_{n}$. Then we have

$$
\begin{aligned}
\left\|v_{n}-x^{*}\right\|^{2}= & \left\|u_{n}+\gamma_{n} A^{*}(V-I) A u_{n}-x^{*}\right\|^{2} \\
= & \left\|u_{n}-x^{*}\right\|^{2}+\gamma_{n}^{2}\left\|A^{*}(V-I) A u_{n}\right\|^{2} \\
& +2 \gamma_{n}\left\langle u_{n}-x^{*}, A^{*}(V-I) A u_{n}\right\rangle .
\end{aligned}
$$

Since $V A x^{*}=A x^{*}$, we get

$$
\begin{aligned}
\left\langle u_{n}-\right. & \left.x^{*}, A^{*}(V-I) A u_{n}\right\rangle \\
= & \left\langle A u_{n}-A x^{*},(V-I) A u_{n}\right\rangle \\
= & \left\langle A u_{n}-A x^{*}+(V-I) A u_{n}-(V-I) A u_{n},(V-I) A u_{n}\right\rangle \\
= & \left\langle V A u_{n}-A x^{*},(V-I) A u_{n}\right\rangle-\left\|(V-I) A u_{n}\right\|^{2} \\
= & \frac{1}{2}\left[\left\|V A u_{n}-A x^{*}\right\|^{2}+\left\|(V-I) A u_{n}\right\|^{2}-\left\|A u_{n}-A x^{*}\right\|^{2}\right] \\
& -\left\|(V-I) A u_{n}\right\|^{2} \\
\leq & \frac{1}{2}\left[\left\|A u_{n}-A x^{*}\right\|^{2}-\left\|A u_{n}-A x^{*}\right\|^{2}\right]-\frac{1}{2}\left\|(V-I) A u_{n}\right\|^{2} \\
= & -\frac{1}{2}\left\|(V-I) A u_{n}\right\|^{2} .
\end{aligned}
$$

From (3.3) and (3.4), we obtain

$$
\left\|v_{n}-x^{*}\right\|^{2} \leq\left\|u_{n}-x^{*}\right\|^{2}-\gamma_{n}\left(\left\|(V-I) A u_{n}\right\|^{2}-\gamma_{n}\left\|A^{*}(V-I) A u_{n}\right\|^{2}\right) .
$$


Now

$$
\begin{aligned}
\left\|x_{n+1}-x^{*}\right\|^{2} & =\left\|U\left(u_{n}+\gamma_{n} A^{*}(V-I) A u_{n}\right)-x^{*}\right\|^{2} \\
& \leq\left\|u_{n}+\gamma_{n} A^{*}(V-I) A u_{n}-x^{*}\right\|^{2} \\
& =\left\|u_{n}-x^{*}\right\|^{2}-\gamma_{n}\left(\left\|(V-I) A u_{n}\right\|^{2}-\gamma_{n}\left\|A^{*}(V-I) A u_{n}\right\|^{2}\right) .
\end{aligned}
$$

From (3.2), (3.3) and (3.4), we get

$$
\begin{aligned}
\left\|x_{n+1}-x^{*}\right\|^{2} \leq & \left(\left\|x_{n}-x^{*}\right\|+\alpha_{n} \tau_{n}\left\|S x^{*}-x^{*}\right\|\right)^{2} \\
& -\gamma_{n}\left(\left\|(V-I) A u_{n}\right\|^{2}-\gamma_{n}\left\|A^{*}(V-I) A u_{n}\right\|^{2}\right) .
\end{aligned}
$$

Now, using the definition of $\gamma_{n}$, we get

$$
\left\|x_{n+1}-x^{*}\right\| \leq\left\|x_{n}-x^{*}\right\|+\alpha_{n} \tau_{n}\left\|S x^{*}-x^{*}\right\| .
$$

Since $\sum_{n=0}^{\infty} \tau_{n}<\infty$, we have $\sum_{n=0}^{\infty} \alpha_{n} \tau_{n}<\infty$. Thus, by using Lemma 2.7 to (3.8), we conclude that $\lim _{n \rightarrow \infty}\left\|x_{n}-x^{*}\right\|$ exists and the value is finite. Hence, $\left\{x_{n}\right\}$ is bounded and so are $\left\{u_{n}\right\}$ and $\left\{v_{n}\right\}$.

Now, from (3.7), we get

$$
\begin{aligned}
\gamma_{n}( & \left.\left\|(V-I) A u_{n}\right\|^{2}-\gamma_{n}\left\|A^{*}(V-I) A u_{n}\right\|^{2}\right) \\
\leq & \left(\left\|x_{n}-x^{*}\right\|+\alpha_{n} \tau_{n}\left\|S x^{*}-x^{*}\right\|\right)^{2}-\left\|x_{n+1}-x^{*}\right\|^{2} \\
= & \left\|x_{n}-x^{*}\right\|^{2}-\left\|x_{n+1}-x^{*}\right\|^{2}+\alpha_{n}^{2} \tau_{n}^{2}\left\|S x^{*}-x^{*}\right\|^{2} \\
& +2 \alpha_{n} \tau_{n}\left\|x_{n}-x^{*}\right\|\left\|S x^{*}-x^{*}\right\| .
\end{aligned}
$$

Since $\lim _{n \rightarrow \infty} \tau_{n}=0$, we get

$$
\gamma_{n}\left(\left\|(V-I) A u_{n}\right\|^{2}-\gamma_{n}\left\|A^{*}(V-I) A u_{n}\right\|^{2}\right) \rightarrow 0 \text { as } n \rightarrow \infty,
$$

which by definition of $\gamma_{n}$, implies that

$$
\frac{\sigma_{n}\left(1-\sigma_{n}\right)\left\|(V-I) A u_{n}\right\|^{4}}{\left\|A^{*}(V-I) A u_{n}\right\|^{2}} \rightarrow 0 \quad \text { as } n \rightarrow \infty .
$$

Since $0<a \leq \sigma_{n} \leq b<1$ and $\left\|A^{*}(V-I) A u_{n}\right\|$ is bounded, we get

$$
\left\|(V-I) A u_{n}\right\| \rightarrow 0 \quad \text { as } n \rightarrow \infty
$$

Now

$$
\left\|A^{*}(V-I) A u_{n}\right\|=\left\|A^{*}\right\|\left\|(V-I) A u_{n}\right\| \rightarrow 0 \quad \text { as } n \rightarrow \infty .
$$

So,

$$
\left\|u_{n}-v_{n}\right\|=\left\|A^{*}(V-I) A u_{n}\right\| \rightarrow 0 \quad \text { as } n \rightarrow \infty .
$$


Now, we estimate

$$
\begin{aligned}
\left\|x_{n+1}-x_{n}\right\|= & \left\|x_{n+1}-x^{*}-\left(x_{n}-x^{*}\right)\right\| \\
= & \left\|x_{n+1}-x^{*}\right\|^{2}-\left\|x_{n}-x^{*}\right\|^{2}-2\left\langle x_{n+1}-x_{n}, x_{n}-x^{*}\right\rangle \\
= & \left\|x_{n+1}-x^{*}\right\|^{2}-\left\|x_{n}-x^{*}\right\|^{2}-2\left\langle x_{n+1}-p, x_{n}-x^{*}\right\rangle \\
& +2\left\langle x_{n}-p, x_{n}-x^{*}\right\rangle,
\end{aligned}
$$

where $p$ is a weak limit point of $\left\{x_{n}\right\}$. Since $\lim _{n \rightarrow \infty}\left\|x_{n}-x^{*}\right\|$ exists, we get

$$
\left\|x_{n+1}-x_{n}\right\| \rightarrow 0 \quad \text { as } n \rightarrow \infty
$$

Since $\liminf _{n \rightarrow \infty} r_{n}>0$, there exists a number $r>0$ such that $r_{n}>r$. Hence, we get

$$
\begin{aligned}
\left\|x_{n+1}-x^{*}\right\|^{2} & =\left\|U v_{n}-U x^{*}\right\|^{2} \\
& =\left\|T_{r_{n}}^{F_{1}}\left(I-r_{n} g_{1}\right) v_{n}-T_{r_{n}}^{F_{1}}\left(I-r_{n} g_{1}\right) x^{*}\right\|^{2} \\
& \leq\left\|\left(I-r_{n} g_{1}\right) v_{n}-\left(I-r_{n} g_{1}\right) x^{*}\right\|^{2} \\
& \leq\left\|v_{n}-x^{*}\right\|^{2}-r_{n}\left(2 \eta_{1}-r_{n}\right)\left\|g_{1} v_{n}-g_{1} x^{*}\right\|^{2} \\
& \leq\left\|v_{n}-x^{*}\right\|^{2}-r\left(2 \eta_{1}-\alpha\right)\left\|g_{1} v_{n}-g_{1} x^{*}\right\|^{2},
\end{aligned}
$$

that is,

$$
\begin{aligned}
r\left(2 \eta_{1}-\alpha\right)\left\|g_{1} v_{n}-g_{1} x^{*}\right\|^{2} \leq & \left\|v_{n}-x^{*}\right\|^{2}-\left\|x_{n+1}-x^{*}\right\|^{2} \\
\leq & \left\|u_{n}-x^{*}\right\|^{2}-\left\|x_{n+1}-x^{*}\right\|^{2} \\
\leq & \left\|x_{n}-x^{*}\right\|^{2}-\left\|x_{n+1}-x^{*}\right\|^{2}+\alpha_{n}^{2} \tau_{n}^{2}\left\|S x^{*}-x^{*}\right\|^{2} \\
& +2 \alpha_{n} \tau_{n}\left\|x_{n}-x^{*}\right\|\left\|S x^{*}-x^{*}\right\| \\
\leq & \left\|x_{n}-x_{n+1}\right\|\left(\left\|x_{n}-x^{*}\right\|+\left\|x_{n+1}-x^{*}\right\|\right) \\
& +\alpha_{n}^{2} \tau_{n}^{2}\left\|S x^{*}-x^{*}\right\|^{2}+2 \alpha_{n} \tau_{n}\left\|x_{n}-x^{*}\right\|\left\|S x^{*}-x^{*}\right\| .
\end{aligned}
$$

Since $\lim _{n \rightarrow \infty} \tau_{n}=0,\left\|x_{n}-x^{*}\right\|$ is bounded and $r\left(2 \eta_{1}-\alpha\right)>0$, we get

$$
\lim _{n \rightarrow \infty}\left\|g_{1} v_{n}-g_{1} x^{*}\right\|=0
$$

Now, from the firmly nonexpansivity of $T_{r_{n}}^{F_{1}}$, we get

$$
\begin{aligned}
\left\|x_{n+1}-x^{*}\right\|^{2} \leq & \left\langle\left(I-r_{n} g_{1}\right) v_{n}-\left(I-r_{n} g_{1}\right) x^{*}, x_{n+1}-x^{*}\right\rangle \\
= & \frac{1}{2}\left[\left\|\left(I-r_{n} g_{1}\right) v_{n}-\left(I-r_{n} g_{1}\right) x^{*}\right\|^{2}+\left\|x_{n+1}-x^{*}\right\|^{2}\right. \\
& \left.-\left\|v_{n}-x_{n+1}-r_{n}\left(g_{1} v_{n}-g_{1} x^{*}\right)\right\|^{2}\right] \\
\leq & \frac{1}{2}\left[\left\|v_{n}-x^{*}\right\|^{2}+\left\|x_{n+1}-x^{*}\right\|^{2}-\left\|v_{n}-x_{n+1}\right\|^{2}\right.
\end{aligned}
$$




$$
\begin{aligned}
& \left.+2 r_{n}\left\langle v_{n}-x_{n+1}, g_{1} v_{n}-g_{1} x^{*}\right\rangle-r_{n}^{2}\left\|g_{1} v_{n}-g_{1} x^{*}\right\|^{2}\right] \\
\leq & \frac{1}{2}\left[\left\|v_{n}-x^{*}\right\|^{2}+\left\|x_{n+1}-x^{*}\right\|^{2}-\left\|v_{n}-x_{n+1}\right\|^{2}\right. \\
& \left.+2 r_{n}\left\|v_{n}-x_{n+1}\right\|\left\|g_{1} v_{n}-g_{1} x^{*}\right\|\right],
\end{aligned}
$$

this implies that

$$
\left\|x_{n+1}-x^{*}\right\|^{2} \leq\left\|v_{n}-x^{*}\right\|^{2}-\left\|v_{n}-x_{n+1}\right\|^{2}+2 r_{n}\left\|v_{n}-x_{n+1}\right\|\left\|g_{1} v_{n}-g_{1} x^{*}\right\| .
$$

That is,

$$
\left\|v_{n}-x_{n+1}\right\|^{2} \leq\left\|v_{n}-x^{*}\right\|^{2}-\left\|x_{n+1}-x^{*}\right\|^{2}+2 r_{n}\left\|v_{n}-x_{n+1}\right\|\left\|g_{1} v_{n}-g_{1} x^{*}\right\| .
$$

Hence, we have

$$
\begin{aligned}
\left\|v_{n}-x_{n+1}\right\|^{2} \leq & \left\|x_{n}-x^{*}\right\|^{2}-\left\|x_{n+1}-x^{*}\right\|^{2}+\alpha_{n}^{2} \tau_{n}^{2}\left\|S x^{*}-x^{*}\right\|^{2} \\
& +2 \alpha_{n} \tau_{n}\left\|x_{n}-x^{*}\right\|\left\|S x^{*}-x^{*}\right\|+2 r_{n}\left\|v_{n}-x_{n+1}\right\|\left\|g_{1} v_{n}-g_{1} x^{*}\right\| \\
\leq & \left\|x_{n}-x_{n+1}\right\|\left(\left\|x_{n}-x^{*}\right\|+\left\|x_{n+1}-x^{*}\right\|\right)+\alpha_{n}^{2} \tau_{n}^{2}\left\|S x^{*}-x^{*}\right\|^{2} \\
& +2 \alpha_{n} \tau_{n}\left\|x_{n}-x^{*}\right\|\left\|S x^{*}-x^{*}\right\|+2 r_{n}\left\|v_{n}-x_{n+1}\right\|\left\|g_{1} v_{n}-g_{1} x^{*}\right\| .
\end{aligned}
$$

Using (3.11) and (3.12), we get

$$
\lim _{n \rightarrow \infty}\left\|v_{n}-x_{n+1}\right\|=0
$$

Now

$$
\left\|x_{n}-v_{n}\right\| \leq\left\|x_{n}-x_{n+1}\right\|+\left\|x_{n+1}-v_{n}\right\| \rightarrow 0 \quad \text { as } n \rightarrow \infty .
$$

Again, by using (3.10) and (3.14), we get

$$
\left\|x_{n}-u_{n}\right\| \leq\left\|x_{n}-v_{n}\right\|+\left\|v_{n}-u_{n}\right\| \rightarrow 0 \quad \text { as } n \rightarrow \infty
$$

Now, we show that $p \in \mathcal{F}$. Since $T_{2}^{n} T_{1}^{n}$ is an averaged mapping, it is nonexpansive. Using the boundedness of $\left\{x_{n}\right\}$ and nonexpansivity of $S$, there exists a $K>0$ such that $\| S x_{n}-$ $T_{2}^{n} T_{1}^{n} x_{n} \| \leq K$ for all $n \geq 0$. Now, we know that

$$
\begin{aligned}
\left\|u_{n}-T_{2}^{n} T_{1}^{n} x_{n}\right\|= & \left\|\left(1-\alpha_{n}\right) x_{n}+\alpha_{n}\left(\tau_{n} S x_{n}+\left(1-\tau_{n}\right) T_{2}^{n} T_{1}^{n} x_{n}\right)-T_{2}^{n} T_{1}^{n} x_{n}\right\| \\
\leq & \left(1-\alpha_{n}\right)\left\|x_{n}-T_{2}^{n} T_{1}^{n} x_{n}\right\|+\alpha_{n} \tau_{n}\left\|S x_{n}-T_{2}^{n} T_{1}^{n} x_{n}\right\| \\
\leq & \left(1-\alpha_{n}\right)\left\|x_{n}-u_{n}\right\|+\left(1-\alpha_{n}\right)\left\|u_{n}-T_{2}^{n} T_{1}^{n} x_{n}\right\| \\
& +\alpha_{n} \tau_{n}\left\|S x_{n}-T_{2}^{n} T_{1}^{n} x_{n}\right\|,
\end{aligned}
$$

this implies that

$$
\begin{aligned}
\alpha_{n}\left\|u_{n}-T_{2}^{n} T_{1}^{n} x_{n}\right\| & \leq\left(1-\alpha_{n}\right)\left\|x_{n}-u_{n}\right\|+\alpha_{n} \tau_{n} K \\
& \leq\left\|x_{n}-u_{n}\right\|+\alpha_{n} \tau_{n} K .
\end{aligned}
$$


Hence, we have

$$
\left\|u_{n}-T_{2}^{n} T_{1}^{n} x_{n}\right\| \leq \frac{\left\|x_{n}-u_{n}\right\|}{\alpha_{n}}+\tau_{n} K
$$

It follows from the conditions (i)-(ii) that

$$
\lim _{n \rightarrow \infty} \frac{\left\|x_{n}-u_{n}\right\|}{\alpha_{n}}=\lim _{n \rightarrow \infty} \tau_{n} \frac{\left\|x_{n}-u_{n}\right\|}{\tau_{n} \alpha_{n}}=0 .
$$

Hence from (3.16), we get

$$
\lim _{n \rightarrow \infty}\left\|u_{n}-T_{2}^{n} T_{1}^{n} x_{n}\right\|=0
$$

Now, by using (3.15), we get

$$
\left\|x_{n}-T_{2}^{n} T_{1}^{n} x_{n}\right\| \leq\left\|x_{n}-u_{n}\right\|+\left\|u_{n}-T_{2}^{n} T_{1}^{n} x_{n}\right\| \rightarrow 0 \quad \text { as } n \rightarrow \infty .
$$

Since $\left\{x_{n}\right\}$ is bounded, there exists a subsequence $\left\{x_{n_{j}}\right\}$ of $\left\{x_{n}\right\}$ such that $x_{n_{j}} \rightarrow p$ as $j \rightarrow \infty$. Noticing that $\left\{\delta_{n}^{i}\right\}$ is bounded for $i=1,2$, we can assume that $\delta_{n_{j}}^{i} \rightarrow \delta_{\infty}^{i}$ as $j \rightarrow \infty$, where $0<\delta_{\infty}^{i}<1$ for $i=1,2$. Define, for $i=1,2$,

$$
T_{i}^{\infty}=\left(1-\delta_{\infty}^{i}\right) I+\delta_{\infty}^{i} P_{C}\left(\beta_{i} I+\left(1-\beta_{i}\right) T_{i}\right)
$$

Now, by Lemma 2.3 and Lemma 2.4, $\operatorname{Fix}\left(P_{C}\left(\beta_{i} I+\left(1-\beta_{i}\right) T_{i}\right)\right)=\operatorname{Fix}\left(T_{i}\right)$. Again, since $P_{C}\left(\beta_{i} I+\left(1-\beta_{i}\right) T_{i}\right)$ is a nonexpansive mapping, $T_{i}^{\infty}$ is averaged and $\operatorname{Fix}\left(T_{i}^{\infty}\right)=\operatorname{Fix}\left(T_{i}\right)$ for $i=1,2$.

Furthermore, since

$$
\operatorname{Fix}\left(T_{1}^{\infty}\right) \cap \operatorname{Fix}\left(T_{2}^{\infty}\right)=\operatorname{Fix}\left(T_{1}\right) \cap \operatorname{Fix}\left(T_{2}\right)=\operatorname{Fix}(\mathcal{S}) \neq \emptyset,
$$

by Lemma 2.2, we get

$$
\operatorname{Fix}\left(T_{2}^{\infty} T_{1}^{\infty}\right)=\operatorname{Fix}\left(T_{1}^{\infty}\right) \cap \operatorname{Fix}\left(T_{2}^{\infty}\right)=\operatorname{Fix}(\mathcal{S})
$$

Note that

$$
\left\|T_{i}^{n_{j}} t-T_{i}^{\infty} t\right\| \leq\left|\delta_{n_{j}}^{i}-\delta_{\infty}^{i}\right|\left(\|t\|+\left\|P_{C}\left(\beta_{i} t+\left(1-\beta_{i}\right) T_{i}(t)\right)\right\|\right) .
$$

Hence, we get

$$
\lim _{j \rightarrow \infty} \sup _{t \in B}\left\|T_{i}^{n_{j}} t-T_{i}^{\infty} t\right\|=0
$$

where $B$ is an arbitrary bounded subset of $H_{1}$. Also, we have

$$
\begin{aligned}
\left\|x_{n_{j}}-T_{2}^{\infty} T_{1}^{\infty} x_{n_{j}}\right\| \leq & \left\|x_{n_{j}}-T_{2}^{n_{j}} T_{1}^{n_{j}} x_{n_{j}}\right\|+\left\|T_{2}^{n_{j}} T_{1}^{n_{j}} x_{n_{j}}-T_{2}^{\infty} T_{1}^{n_{j}} x_{n_{j}}\right\| \\
& +\left\|T_{2}^{\infty} T_{1}^{n_{j}} x_{n_{j}}-T_{2}^{\infty} T_{1}^{\infty} x_{n_{j}}\right\|
\end{aligned}
$$




$$
\begin{aligned}
\leq & \left\|x_{n_{j}}-T_{2}^{n_{j}} T_{1}^{n_{j}} x_{n_{j}}\right\|+\left\|T_{2}^{n_{j}} T_{1}^{n_{j}} x_{n_{j}}-T_{2}^{\infty} T_{1}^{n_{j}} x_{n_{j}}\right\| \\
& +\left\|T_{1}^{n_{j}} x_{n_{j}}-T_{1}^{\infty} x_{n_{j}}\right\| \\
\leq & \left\|x_{n_{j}}-T_{2}^{n_{j}} T_{1}^{n_{j}} x_{n_{j}}\right\|+\sup _{t \in B_{1}}\left\|T_{2}^{n_{j}} t-T_{2}^{\infty} t\right\| \\
& +\sup _{t \in B_{2}}\left\|T_{1}^{n_{j}} t-T_{1}^{\infty} t\right\|,
\end{aligned}
$$

where $B_{1}$ is a bounded subset including $\left\{T_{1}^{n_{j}} x_{n_{j}}\right\}$ and $B_{2}$ is a bounded subset including $\left\{x_{n_{j}}\right\}$. It follows from (3.17), (3.18) and (3.19) that

$$
\lim _{j \rightarrow \infty}\left\|x_{n_{j}}-T_{2}^{\infty} T_{1}^{\infty} x_{n_{j}}\right\|=0
$$

Hence, from Lemma 2.5, we get $p \in \operatorname{Fix}\left(T_{2}^{\infty} T_{1}^{\infty}\right)=\operatorname{Fix}\left(T_{1}\right) \cap \operatorname{Fix}\left(T_{2}\right)$.

Now, we show that $x^{*} \in \mathcal{S}$. It follows from (3.1) that

$$
u_{n}-x_{n}=\alpha_{n}\left(\tau_{n}\left(S x_{n}-x_{n}\right)+\left(1-\tau_{n}\right)\left(T_{2}^{n} T_{1}^{n} x_{n}-x_{n}\right)\right),
$$

and hence,

$$
\frac{1}{\alpha_{n} \tau_{n}}\left(x_{n}-u_{n}\right)=\left((I-S) x_{n}+\left(\frac{1-\tau_{n}}{\tau_{n}}\right)\left(I-T_{2}^{n} T_{1}^{n}\right) x_{n}\right) .
$$

By using Lemma 2.9(i), it follows that the operator sequence $\left\{\left(\frac{1-\tau_{n}}{\tau_{n}}\right)\left(I-T_{2}^{n} T_{1}^{n}\right)\right\}$ is graph convergent to $N_{\mathrm{Fix}\left(T_{1}\right) \cap \mathrm{Fix}\left(T_{2}\right)}$, and hence, from Lemma 2.9(ii), it follows that the opera-

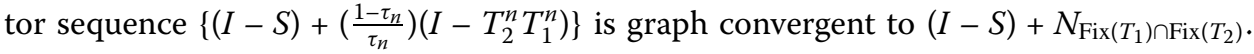
Now, by replacing $n$ by $n_{j}$ and letting the limit in (3.20) and considering the fact that $\lim _{n \rightarrow \infty} \frac{1}{\alpha_{n} \tau_{n}}\left\|x_{n}-u_{n}\right\|=0$ and the graph of $(I-S)+N_{\mathrm{Fix}\left(T_{1}\right) \cap \mathrm{Fix}\left(T_{2}\right)}$ is weakly-strongly closed, we get

$$
0 \in(I-S) p+N_{\mathrm{Fix}\left(T_{1}\right) \cap \operatorname{Fix}\left(T_{2}\right) p}
$$

that is, $p \in \mathcal{S}$.

We now show that $p \in \Gamma$. Since $u_{n}=U\left(v_{n}\right)=T_{r_{n}}^{F_{1}}\left(I-r_{n} g_{1}\right)\left(v_{n}\right)$, we have

$$
F_{1}\left(x_{n+1}, y\right)+\left\langle g_{1} v_{n}, y-x_{n+1}\right\rangle+\frac{1}{r_{n}}\left\langle y-x_{n+1}, x_{n+1}-v_{n}\right\rangle \geq 0, \quad \forall y \in C
$$

From the monotonicity of $F_{1}$, we get

$$
\left\langle g_{1} v_{n}, y-x_{n+1}\right\rangle+\frac{1}{r_{n}}\left\langle y-x_{n+1}, x_{n+1}-v_{n}\right\rangle \geq F_{1}\left(y, x_{n+1}\right), \quad \forall y \in C .
$$

Replacing $n$ with $n_{j}$ in the above inequality, we get

$$
\left\langle g_{1} v_{n_{j}}, y-x_{n_{j}+1}\right\rangle+\frac{1}{r_{n_{j}}}\left\langle y-x_{n_{j}+1}, x_{n_{j}+1}-v_{n_{j}}\right\rangle \geq F_{1}\left(y, x_{n_{j}+1}\right), \quad \forall y \in C .
$$


For $t$ with $0<t \leq 1$ and $y \in C$, let $y_{t}=t y+(1-t) p$. Since $y \in C$ and $p \in C$, we get $y_{t} \in C$ and hence $F_{1}\left(y_{t}, p\right) \leq 0$. So, from the above inequality, we get

$$
\begin{aligned}
\left\langle y_{t}-x_{n+1}, g_{1} y_{t}\right\rangle \geq & \left\langle y_{t}-x_{n+1}, g_{1} y_{t}\right\rangle-\left\langle y_{t}-x_{n+1}, g_{1} v_{t}\right\rangle \\
& -\frac{1}{r_{n_{j}}}\left\langle y_{t}-x_{n_{j}+1}, \frac{x_{n_{j}+1}-v_{n_{j}}}{r_{n_{j}}}\right\rangle+F_{1}\left(y_{t}, x_{n_{j}+1}\right) \\
= & \left\langle y_{t}-x_{n+1}, g_{1} y_{t}-g_{1} x_{n_{j}+1}\right\rangle+\left\langle y_{t}-x_{n_{j}+1}, g_{1} x_{n_{j}+1}-g_{1} v_{t}\right\rangle \\
& -\frac{1}{r_{n_{j}}}\left\langle y_{t}-x_{n_{j}+1}, \frac{x_{n_{j}+1}-v_{n_{j}}}{r_{n_{j}}}\right\rangle+F_{1}\left(y_{t}, x_{n_{j}+1}\right) .
\end{aligned}
$$

Since $\left\{x_{n}\right\},\left\{u_{n}\right\}$ and $\left\{v_{n}\right\}$ have the same asymptotic behavior and $x_{n_{j}} \rightarrow p$, there exist subsequences $\left\{u_{n_{j}}\right\}$ of $\left\{u_{n}\right\}$ and $\left\{v_{n_{j}}\right\}$ of $\left\{v_{n}\right\}$ such that $u_{n_{j}} \rightarrow p$ and $v_{n_{j}} \rightarrow p$. Since $\lim _{j \rightarrow \infty}\left\|x_{n_{j}+1}-v_{n_{j}}\right\|=0$ and $f_{1}$ is Lipschitz continuous, we have $\lim _{j \rightarrow \infty}\left\|g_{1} x_{n_{j}+1}-g_{1} v_{n_{j}}\right\|=0$. Since $\liminf _{n \rightarrow \infty} r_{n}>0$, there exists a number $r>0$ such that $\liminf _{n \rightarrow \infty} r_{n}=r$. Hence, we have

$$
\begin{aligned}
\lim _{j \rightarrow \infty} \frac{\left\|x_{n_{j}+1}-v_{n_{j}}\right\|}{r_{n_{j}}} & \leq \frac{\lim _{j \rightarrow \infty}\left\|x_{n_{j}+1}-v_{n_{j}}\right\|}{\liminf \operatorname{in}_{n \rightarrow \infty} r_{n_{j}}} \\
& =\frac{1}{r} \lim _{j \rightarrow \infty}\left\|x_{n_{j}+1}-v_{n_{j}}\right\| \\
& =0 .
\end{aligned}
$$

Furthermore, from the monotonicity of $g_{1}$ and lower semicontinuity of $F_{1}$, we have

$$
\left\langle y_{t}-p, g_{1} y_{t}\right\rangle \geq F_{1}\left(y_{t}, p\right)
$$

as $j \rightarrow \infty$. And also, from the convexity of $F_{1}$, we have

$$
\begin{aligned}
0 & =F_{1}\left(y_{t}, y_{t}\right) \\
& \leq t F_{1}\left(y_{t}, y\right)+(1-t)\left\langle y_{t}-p, g_{1} y_{t}\right\rangle \\
& \leq t F_{1}\left(y_{t}, y\right)+(1-t) t\left\langle y-p, g_{1} y_{t}\right\rangle .
\end{aligned}
$$

Hence, $0 \leq F_{1}\left(y_{t}, y\right)+(1-t)\left\langle y-p, g_{1} y_{t}\right\rangle$. Letting $t \rightarrow 0_{+}$, for each $y \in C$, we have

$$
F_{1}(p, y)+\left\langle y-p, g_{1} p\right\rangle \geq 0
$$

This implies that $p \in \operatorname{Sol}(\operatorname{MEP}(1.9))$.

Now, we need to show that $A p \in \operatorname{Sol}(\operatorname{MEP}(1.10))$. Since $A$ is bounded linear operator, we have $A x_{n_{j}} \rightarrow A p$. Now, setting $d_{n_{j}}=A u_{n_{j}}-V A u_{n_{j}}$, we get $d_{n_{j}} \rightarrow 0$ and $A u_{n_{j}}-d_{n_{j}}=V A u_{n_{j}}$. Therefore, from Lemma 2.6, we have

$$
\begin{aligned}
& F_{2}\left(A u_{n_{j}}-d_{n_{j}}, z\right)+\left\langle g_{2} A u_{n_{j}}, z-\left(A u_{n_{j}}-d_{n_{j}}\right)\right\rangle \\
& \quad+\frac{1}{r_{n_{j}}}\left\langle z-\left(A u_{n_{j}}-d_{n_{j}}\right), A u_{n_{j}}-d_{n_{j}}-A u_{n_{j}}\right\rangle \geq 0, \quad \forall z \in D .
\end{aligned}
$$


Since $F_{2}$ is upper semicontinuous in the first argument, taking lim sup to the above inequality as $j \rightarrow \infty$ and using $\liminf _{n \rightarrow \infty} r_{n}>0$, we get

$$
F_{2}(A p, z)+\left\langle z-A p, g_{2} A p\right\rangle \geq 0, \quad \forall z \in D
$$

which implies that $A p \in \operatorname{Sol}(\operatorname{MEP}(1.10))$. This shows that $p \in \Gamma$ and thus $p \in \mathcal{F}$. It follows from the existence of $\lim _{n \rightarrow \infty}\left\|x_{n}-p\right\|$ and the fact that Hilbert space satisfies Opial's conditions, the sequence $\left\{x_{n}\right\}$ has only one weak limit point, and hence $\left\{x_{n}\right\}$ converges weakly to $p \in \mathcal{F}$.

The following consequence is a weak convergence theorem for computing a common solution of a mixed equilibrium problem and a hierarchical fixed point problem in a real Hilbert space.

Corollary 3.2 Let $H_{1}$ be a Hilbert spaces. Let $C$ be a nonempty closed and convex subset of $H_{1}$. Suppose $F_{1}: C \times C \rightarrow \mathbb{R}$ is a bifunction which satisfy Assumption A. Let $g_{1}: C \rightarrow H_{1}$ be $\eta_{1}$-inverse strongly monotone mapping. Let $S: C \rightarrow C$ be a nonexpansive self-mapping and $\left\{T_{i}\right\}_{i=1}^{N}: C \rightarrow H_{1}$ be $k_{i}$-strictly pseudocontractive nonself-mappings. Assume that $\mathcal{F}=$ $\operatorname{MEP}\left(F_{1}, g_{1}\right) \cap \mathcal{S} \neq \emptyset$. Define a sequence $\left\{x_{n}\right\}$ as follows:

$$
\left\{\begin{array}{l}
x_{0} \in C, \\
u_{n}=\left(1-\alpha_{n}\right) x_{n}+\alpha_{n}\left(\tau_{n} S x_{n}+\left(1-\tau_{n}\right) T_{N}^{n} T_{N-1}^{n} \cdots T_{1}^{n} x_{n}\right), \\
x_{n+1}=T_{r_{n}}^{F_{1}}\left(I-r_{n} g_{1}\right)\left(u_{n}\right), \quad n \geq 1,
\end{array}\right.
$$

where $T_{i}^{n}=\left(1-\delta_{n}^{i}\right) I+\delta_{n}^{i} P_{C}\left(\beta_{i} I+\left(1-\beta_{i}\right) T_{i}\right), 0 \leq k_{i} \leq \beta_{i}<1, \delta_{n}^{i} \in(0,1)$ for $i=1,2, \ldots, N$. Let $\left\{\alpha_{n}\right\},\left\{\tau_{n}\right\}$ be two real sequences in $(0,1)$ and $\left\{r_{n}\right\} \subset\left(0,2 \eta_{1}\right)$. Suppose the following conditions (i)-(iv) of Theorem 3.1 are satisfied. Then the sequence $\left\{x_{n}\right\}$ converges weakly to $x^{*} \in \mathcal{F}$

Proof Taking $A=0$ in Theorem 3.1, the conclusion of Corollary 3.2 is followed.

In the above theorem, the sequence generated by algorithm (3.1) converges weakly to a common solution of a split mixed equilibrium problem and a hierarchical fixed point problem.

In this section, our other aim is to prove a strong convergence theorem for a hierarchical fixed point problem and split equilibrium problem for some special cases.

We consider the following hierarchical fixed point and split equilibrium problem: Find $x^{*} \in \Gamma \cap \mathcal{S}$ such that

$$
\left\langle x^{*}-S x^{*}, x^{*}-y\right\rangle \leq 0, \quad \forall y \in \Gamma \cap \mathcal{S},
$$

where $\mathcal{S}=\bigcap_{i=1}^{N} \operatorname{Fix}\left(T_{i}\right)$ and $\Gamma$ is the solution set of the split equilibrium problem (1.5)(1.6). When $S$ is a contraction mapping, a special case of nonexpansive mappings, we prove a strong convergence theorem for the above problem. We need the following results for our study. 
Lemma 3.3 ([8]) Let $F_{1}: C \times C \rightarrow \mathbb{R}$ be a bifunction satisfying Assumption $\mathrm{A}$ and $T_{r}^{F_{1}}$ be defined as in Lemma 2.6. Let $x, y \in H_{1}$ and $r_{1}, r_{2}>0$. Then

$$
\left\|T_{r_{2}}^{F_{1}} y-T_{r_{1}}^{F_{1}} x\right\| \leq\|y-x\|+\left|\frac{r_{2}-r_{1}}{r_{2}}\right|\left\|T_{r_{2}}^{F_{1}} y-y\right\|
$$

Lemma 3.4 ([26]) Let $\left\{x_{n}\right\}$ and $\left\{z_{n}\right\}$ be bounded sequences in a Banach space X. Let $\left\{\beta_{n}\right\}$ be a sequence in $[0,1]$ which satisfies the condition $0<\liminf _{n \rightarrow \infty} \beta_{n} \leq \lim _{\sup _{n \rightarrow \infty}} \beta_{n}<1$. Suppose $x_{n+1}=\left(1-\beta_{n}\right) z_{n}+\beta_{n} x_{n}$ for all integer $n \geq 0$ and $\limsup _{n \rightarrow \infty}\left(\left\|z_{n+1}-z_{n}\right\|-\| x_{n+1}-\right.$ $\left.x_{n} \|\right) \leq 0$. Then $\lim _{n \rightarrow \infty}\left\|x_{n}-z_{n}\right\|=0$.

Lemma 3.5 ([31]) Let $\left\{\alpha_{n}\right\}$ be a sequence of non-negative real numbers such that

$$
\alpha_{n+1} \leq\left(1-\gamma_{n}\right) \alpha_{n}+\delta_{n}
$$

where $\left\{\gamma_{n}\right\}$ is a sequence in $(0,1)$ and $\left\{\delta_{n}\right\}$ is a sequence such that

(i) $\sum_{n=1}^{\infty} \gamma_{n}=\infty$;

(ii) $\lim \sup _{n \rightarrow \infty} \frac{\delta_{n}}{\gamma_{n}} \leq 0$ or $\sum_{n=1}^{\infty}\left|\delta_{n}\right|<\infty$.

Then $\lim _{n \rightarrow \infty} \alpha_{n}=0$.

Now we are in a position to state our second main result for strong convergence.

Theorem 3.6 Let $H_{1}$ and $H_{2}$ be two Hilbert spaces. Let $C$ and $D$ be nonempty closed and convex subset of $H_{1}$ and $H_{2}$, respectively. Let $A: H_{1} \rightarrow H_{2}$ be a bounded linear operator. Let $F_{1}: C \times C \rightarrow \mathbb{R}$ and $F_{2}: D \times D \rightarrow \mathbb{R}$ be two bifunctions satisfying Assumption $\mathrm{A}$ and $F_{2}$ be upper semicontinuous. Let $S: C \rightarrow C$ be a contraction mapping with coefficient $\rho \geq 0$ and $\left\{T_{i}\right\}_{i=1}^{N}: C \rightarrow H_{1}$ be $k_{i}$-strictly pseudocontractive nonself-mappings. Assume that the solution set of problem (3.22) is nonempty. Define a sequence $\left\{x_{n}\right\}$ as follows:

$$
\left\{\begin{array}{l}
x_{0} \in C \\
u_{n}=U\left(x_{n}+\gamma A^{*}(V-I) A x_{n}\right), \quad n \geq 1, \\
x_{n+1}=\left(1-\alpha_{n}\right) x_{n}+\alpha_{n}\left(\tau_{n} S x_{n}+\left(1-\tau_{n}\right) T_{N}^{n} T_{N-1}^{n} \cdots T_{1}^{n} u_{n}\right),
\end{array}\right.
$$

where $U=T_{r_{n}}^{F_{1}}, V=T_{r_{n}}^{F_{2}}, T_{i}^{n}=\left(1-\delta_{n}^{i}\right) I+\delta_{n}^{i} P_{C}\left(\beta_{i} I+\left(1-\beta_{i}\right) T_{i}\right), 0 \leq k_{i} \leq \beta_{i}<1$, and $\delta_{n}^{i} \in(0,1)$ for $i=1,2, \ldots, N$. Also let $\gamma \in\left(0, \frac{1}{L}\right)$ where $L$ is the spectral radius of the operator $A^{*} A$ and $A^{*}$ is the adjoint operator of $A$. Let $\left\{\alpha_{n}\right\}$ and $\left\{\tau_{n}\right\}$ be two real sequences in $(0,1)$. Suppose the following conditions are satisfied:

(i) $\liminf _{n \rightarrow \infty} r_{n}>0$ and $\lim _{n \rightarrow \infty}\left|r_{n+1}-r_{n}\right|=0$;

(ii) $0 \leq \alpha_{n} \leq b<1$ for some $b \in(0,1)$ and $0<\liminf _{n \rightarrow \infty} \alpha_{n} \leq \limsup _{n \rightarrow \infty} \alpha_{n}<1$;

(iii) $\lim _{n \rightarrow \infty} \tau_{n}=0$ and $\sum_{n=0}^{\infty} \tau_{n}=\infty$;

(iv) $\lim _{n \rightarrow \infty}\left|\delta_{n+1}^{i}-\delta_{n}^{i}\right|=0$ for $i=1,2, \ldots, N$.

Then the sequence $\left\{x_{n}\right\}$ converges strongly to $p \in \Gamma \cap \mathcal{S}$, which is the unique solution of the following variational inequality:

$$
\langle p-S p, p-y\rangle \leq 0, \quad \forall y \in \Gamma \cap \mathcal{S} .
$$


Proof We will prove the theorem for $N=2$. Then our method can be easily extended to the general case. First we show that the sequence $\left\{x_{n}\right\}$ is bounded.

Let $x^{*}$ be a solution of problem (3.22). Then $T_{r_{n}}^{F_{1}} x^{*}=x^{*}$ and $T_{r_{n}}^{F_{2}} A x^{*}=A x^{*}$. So, by (3.23) we get

$$
\begin{aligned}
\left\|u_{n}-x^{*}\right\|^{2} & =\left\|T_{r_{n}}^{F_{1}}\left(x_{n}+\gamma A^{*}\left(T_{r_{n}}^{F_{2}}-I\right) A x_{n}\right)-x^{*}\right\|^{2} \\
& =\left\|T_{r_{n}}^{F_{1}}\left(x_{n}+\gamma A^{*}\left(T_{r_{n}}^{F_{2}}-I\right) A x_{n}\right)-T_{r_{n}}^{F_{1}} x^{*}\right\|^{2} \\
& \leq\left\|x_{n}+\gamma A^{*}\left(T_{r_{n}}^{F_{2}}-I\right) A x_{n}-x^{*}\right\| \\
& \leq\left\|x_{n}-x^{*}\right\|^{2}+\gamma^{2}\left\|A^{*}\left(T_{r_{n}}^{F_{2}}-I\right) A x_{n}\right\|^{2} \\
& +2 \gamma\left(x_{n}-x^{*}, A^{*}\left(T_{r_{n}}^{F_{2}}-I\right) A x_{n}\right\rangle .
\end{aligned}
$$

Then

$$
\begin{aligned}
\left\|u_{n}-x^{*}\right\|^{2} \leq & \left\|x_{n}-x^{*}\right\|^{2}+\gamma^{2}\left\langle\left(T_{r_{n}}^{F_{2}}-I\right) A x_{n}, A^{*} A\left(T_{r_{n}}^{F_{2}}-I\right) A x_{n}\right\rangle \\
& +2 \gamma\left\langle x_{n}-x^{*}, A^{*}\left(T_{r_{n}}^{F_{2}}-I\right) A x_{n}\right\rangle .
\end{aligned}
$$

Now, we have

$$
\begin{aligned}
\gamma^{2}\left\langle\left(T_{r_{n}}^{F_{2}}-I\right) A x_{n}, A^{*} A\left(T_{r_{n}}^{F_{2}}-I\right) A x_{n}\right\rangle & \leq L \gamma^{2}\left\langle\left(T_{r_{n}}^{F_{2}}-I\right) A x_{n},\left(T_{r_{n}}^{F_{2}}-I\right) A x_{n}\right\rangle \\
& =L \gamma^{2}\left\|\left(T_{r_{n}}^{F_{2}}-I\right) A x_{n}\right\|^{2} .
\end{aligned}
$$

Denoting $\Lambda:=2 \gamma\left\langle x_{n}-x^{*}, A^{*}\left(T_{r_{n}}^{F_{2}}-I\right) A x_{n}\right\rangle$ and using (2.3), we have

$$
\begin{aligned}
\Lambda & =2 \gamma\left\langle x_{n}-x^{*}, A^{*}\left(T_{r_{n}}^{F_{2}}-I\right) A x_{n}\right\rangle \\
& =2 \gamma\left\langle A\left(x_{n}-x^{*}\right),\left(T_{r_{n}}^{F_{2}}-I\right) A x_{n}\right\rangle \\
& =2 \gamma\left\langle A\left(x_{n}-x^{*}\right)+\left(T_{r_{n}}^{F_{2}}-I\right) A x_{n}-\left(T_{r_{n}}^{F_{2}}-I\right) A x_{n},\left(T_{r_{n}}^{F_{2}}-I\right) A x_{n}\right\rangle \\
& =2 \gamma\left\{\left\langle\left(T_{r_{n}}^{F_{2}}-I\right) A x^{*},\left(T_{r_{n}}^{F_{2}}-I\right) A x_{n}\right\rangle-\left\|\left(T_{r_{n}}^{F_{2}}-I\right) A x_{n}\right\|^{2}\right\} \\
& =2 \gamma\left\{\frac{1}{2}\left\|\left(T_{r_{n}}^{F_{2}}-I\right) A x_{n}\right\|^{2}-\left\|\left(T_{r_{n}}^{F_{2}}-I\right) A x_{n}\right\|^{2}\right\} \\
& =-\gamma\left\|\left(T_{r_{n}}^{F_{2}}-I\right) A x_{n}\right\|^{2} .
\end{aligned}
$$

Using (3.26), (3.27) and (3.28), we get

$$
\left\|u_{n}-x^{*}\right\|^{2} \leq\left\|x_{n}-x^{*}\right\|^{2}+\gamma(L \gamma-1)\left\|\left(T_{r_{n}}^{F_{2}}-I\right) A x_{n}\right\|^{2} .
$$

From the definition of $\gamma$, we obtain

$$
\left\|u_{n}-x^{*}\right\| \leq\left\|x_{n}-x^{*}\right\|
$$

Now

$$
\begin{aligned}
& \left\|x_{n+1}-x^{*}\right\| \\
& \quad=\left\|\left(1-\alpha_{n}\right)\left(x_{n}-x^{*}\right)+\alpha_{n}\left[\tau_{n}\left(S x_{n}-x^{*}\right)+\left(1-\tau_{n}\right)\left(T_{2}^{n} T_{1}^{n} u_{n}-x^{*}\right)\right]\right\|
\end{aligned}
$$




$$
\begin{aligned}
\leq & \left(1-\alpha_{n}\right)\left\|x_{n}-x^{*}\right\|+\alpha_{n}\left[\tau_{n}\left\|S x_{n}-x^{*}\right\|+\left(1-\tau_{n}\right)\left\|T_{2}^{n} T_{1}^{n} u_{n}-x^{*}\right\|\right] \\
\leq & \left(1-\alpha_{n}\right)\left\|x_{n}-x^{*}\right\|+\alpha_{n}\left[\tau_{n}\left\|S x_{n}-S x^{*}\right\|+\left(1-\tau_{n}\right)\left\|u_{n}-x^{*}\right\|\right] \\
& +\alpha_{n} \tau_{n}\left\|S x^{*}-x^{*}\right\| \\
\leq & \left(1-\alpha_{n}\right)\left\|x_{n}-x^{*}\right\|+\alpha_{n}\left[\tau_{n} \rho\left\|x_{n}-x^{*}\right\|+\left(1-\tau_{n}\right)\left\|x_{n}-x^{*}\right\|\right] \\
& +\alpha_{n} \tau_{n}\left\|S x^{*}-x^{*}\right\| \\
\leq & \left(1-(1-\rho) \alpha_{n} \tau_{n}\right)\left\|x_{n}-x^{*}\right\|+\alpha_{n} \tau_{n}\left\|S x^{*}-x^{*}\right\| \\
\leq & \max \left\{\left\|x_{n}-x^{*}\right\|, \frac{\left\|S x^{*}-x^{*}\right\|}{(1-\rho)}\right\} \\
\leq & \cdots \leq \max \left\{\left\|x_{0}-x^{*}\right\|, \frac{\left\|S x^{*}-x^{*}\right\|}{(1-\rho)}\right\} .
\end{aligned}
$$

Hence the sequence $\left\{x_{n}\right\}$ is bounded.

Now, we show that

$$
\lim _{x \rightarrow \infty}\left\|x_{n+1}-x_{n}\right\|=0
$$

Let us consider $y_{n}=\tau_{n} S x_{n}+\left(1-\tau_{n}\right) T_{2}^{n} T_{1}^{n} u_{n}$. Then

$$
\begin{aligned}
\left\|y_{n+1}-y_{n}\right\| \leq & \tau_{n+1}\left\|S x_{n+1}-S x_{n}\right\|+\left|\tau_{n+1}-\tau_{n}\right|\left\|S x_{n}-T_{2}^{n} T_{1}^{n} u_{n}\right\| \\
& +\left(1-\tau_{n+1}\right)\left\|T_{2}^{n+1} T_{1}^{n+1} u_{n+1}-T_{2}^{n} T_{1}^{n} u_{n}\right\| .
\end{aligned}
$$

In addition, we have

$$
\begin{aligned}
\left\|T_{2}^{n+1} T_{1}^{n+1} u_{n+1}-T_{2}^{n} T_{1}^{n} u_{n}\right\| \\
\leq\left\|T_{2}^{n+1} T_{1}^{n+1} u_{n+1}-T_{2}^{n} T_{1}^{n} u_{n+1}\right\|+\left\|T_{2}^{n} T_{1}^{n} u_{n+1}-T_{2}^{n} T_{1}^{n} u_{n}\right\| \\
\leq\left\|T_{2}^{n+1} T_{1}^{n+1} u_{n+1}-T_{2}^{n+1} T_{1}^{n} u_{n+1}\right\| \\
\quad+\left\|T_{2}^{n+1} T_{1}^{n} u_{n+1}-T_{2}^{n} T_{1}^{n} u_{n+1}\right\|+\left\|u_{n+1}-u_{n}\right\| \\
\leq\left\|T_{1}^{n+1} u_{n+1}-T_{1}^{n} u_{n+1}\right\|+\left\|T_{2}^{n+1} T_{1}^{n} u_{n+1}-T_{2}^{n} T_{1}^{n} u_{n+1}\right\| \\
\quad+\left\|u_{n+1}-u_{n}\right\| .
\end{aligned}
$$

It follows from the definition $T_{i}^{n}$ that

$$
\begin{aligned}
\| & T_{1}^{n+1} u_{n+1}-T_{1}^{n} u_{n+1} \| \\
= & \|\left(1-\delta_{n+1}^{1}\right) u_{n+1}+\delta_{n+1}^{1} P_{C}\left(\beta_{1} I+\left(1-\beta_{1}\right) T_{1}\right) u_{n+1} \\
& \quad-\left(1-\delta_{n}^{1}\right) u_{n+1}+\delta_{n}^{1} P_{C}\left(\beta_{1} I+\left(1-\beta_{1}\right) T_{1}\right) u_{n+1} \| \\
& \leq\left|\delta_{n+1}^{1}-\delta_{n}^{1}\right|\left(\left\|u_{n+1}\right\|+\left\|P_{C}\left(\beta_{1} I+\left(1-\beta_{1}\right) T_{1}\right) u_{n+1}\right\|\right) .
\end{aligned}
$$

Since $\lim _{n \rightarrow+\infty}\left|\delta_{n+1}^{1}-\delta_{n}^{1}\right|=0$, and $\left\{u_{n}\right\},\left\{P_{C}\left(\beta_{1} I+\left(1-\beta_{1}\right) T_{1}\right) u_{n}\right\}$ are bounded, we get

$$
\lim _{n \rightarrow+\infty}\left\|T_{1}^{n+1} u_{n+1}-T_{1}^{n} u_{n+1}\right\|=0
$$


Similarly, we have

$$
\begin{aligned}
& \left\|T_{2}^{n+1} T_{1}^{n} u_{n+1}-T_{2}^{n} T_{1}^{n} u_{n+1}\right\| \\
& \quad \leq\left|\delta_{n+1}^{2}-\delta_{n}^{2}\right|\left(\left\|T_{1}^{n} u_{n+1}\right\|+\left\|P_{C}\left(\beta_{2} I+\left(1-\beta_{2}\right) T_{2}\right) T_{1}^{n} u_{n+1}\right\|\right),
\end{aligned}
$$

from which it follows that

$$
\lim _{n \rightarrow+\infty}\left\|T_{2}^{n+1} T_{1}^{n+1} u_{n+1}-T_{2}^{n} T_{1}^{n} u_{n+1}\right\|=0 .
$$

Since

$$
u_{n}=T_{r_{n}}^{F_{1}}\left(x_{n}+\gamma A^{*}\left(T_{r_{n}}^{F_{2}}-I\right) A x_{n}\right)
$$

and

$$
u_{n+1}=T_{r_{n+1}}^{F_{1}}\left(x_{n+1}+\gamma A^{*}\left(T_{r_{n+1}}^{F_{2}}-I\right) A x_{n+1}\right) \text {, }
$$

it follows from Lemma 3.3 that

$$
\begin{aligned}
\| u_{n+1}- & u_{n} \| \\
= & \left\|T_{r_{n+1}}^{F_{1}}\left(x_{n+1}+\gamma A^{*}\left(T_{r_{n+1}}^{F_{2}}-I\right) A x_{n+1}\right)-T_{r_{n}}^{F_{1}}\left(x_{n}+\gamma A^{*}\left(T_{r_{n}}^{F_{2}}-I\right) A x_{n}\right)\right\| \\
\leq & \left\|T_{r_{n+1}}^{F_{1}}\left(x_{n+1}+\gamma A^{*}\left(T_{r_{n+1}}^{F_{2}}-I\right) A x_{n+1}\right)-T_{r_{n+1}}^{F_{1}}\left(x_{n}+\gamma A^{*}\left(T_{r_{n}}^{F_{2}}-I\right) A x_{n}\right)\right\| \\
& +\left\|T_{r_{n+1}}^{F_{1}}\left(x_{n}+\gamma A^{*}\left(T_{r_{n}}^{F_{2}}-I\right) A x_{n}\right)-T_{r_{n}}^{F_{1}}\left(x_{n}+\gamma A^{*}\left(T_{r_{n}}^{F_{2}}-I\right) A x_{n}\right)\right\| \\
\leq & \left\|\left(x_{n+1}+\gamma A^{*}\left(T_{r_{n+1}}^{F_{2}}-I\right) A x_{n+1}\right)-\left(x_{n}+\gamma A^{*}\left(T_{r_{n}}^{F_{2}}-I\right) A x_{n}\right)\right\| \\
& +\left|\frac{r_{n+1}-r_{n}}{r_{n+1}}\right|\left\|T_{r_{n+1}}^{F_{1}}\left(x_{n}+\gamma A^{*}\left(T_{r_{n}}^{F_{2}}-I\right) A x_{n}\right)-\left(x_{n}+\gamma A^{*}\left(T_{r_{n}}^{F_{2}}-I\right) A x_{n}\right)\right\| \\
\leq & \left\|x_{n+1}-x_{n}-\gamma A^{*} A\left(x_{n+1}-x_{n}\right)\right\|+\gamma\|A\|\left\|T_{r_{n+1}}^{F_{2}} A x_{n+1}-T_{r_{n}}^{F_{2}} A x_{n}\right\|+\delta_{n} \\
\leq & \left\{\left\|x_{n+1}-x_{n}\right\|^{2}-2 \gamma\left\|A x_{n+1}-A x_{n}\right\|^{2}+\gamma^{2}\|A\|^{4}\left\|x_{n+1}-x_{n}\right\|^{2}\right\}^{\frac{1}{2}} \\
& +\gamma\|A\|\left\{\left\|A x_{n+1}-A x_{n}\right\|+\left|\frac{r_{n+1}-r_{n}}{r_{n+1}}\right|\left\|T_{r_{n+1}}^{F_{2}} A x_{n+1}-A x_{n}\right\|\right\}+\delta_{n} \\
\leq & \left(1-2 \gamma\|A\|^{2}+\gamma^{2}\|A\|^{4}\right)^{\frac{1}{2}}\left\|x_{n+1}-x_{n}\right\|+\gamma\|A\|^{2}\left\|x_{n+1}-x_{n}\right\|+\gamma\|A\| \sigma_{n}+\delta_{n} \\
= & \left(1-\gamma\|A\|^{2}\right)\left\|x_{n+1}-x_{n}\right\|+\gamma\|A\|^{2}\left\|x_{n+1}-x_{n}\right\|+\gamma\|A\| \sigma_{n}+\delta_{n} \\
= & \left\|x_{n+1}-x_{n}\right\|+\gamma\|A\| \sigma_{n}+\delta_{n},
\end{aligned}
$$

where

$$
\sigma_{n}=\left|\frac{r_{n+1}-r_{n}}{r_{n+1}}\right|\left\|T_{r_{n+1}}^{F_{2}} A x_{n+1}-A x_{n}\right\|
$$

and

$$
\delta_{n}=\left|\frac{r_{n+1}-r_{n}}{r_{n+1}}\right|\left\|T_{r_{n+1}}^{F_{1}}\left(x_{n}+\gamma A^{*}\left(T_{r_{n}}^{F_{2}}-I\right) A x_{n}\right)-\left(x_{n}+\gamma A^{*}\left(T_{r_{n}}^{F_{2}}-I\right) A x_{n}\right)\right\| .
$$


Hence, using (3.34), (3.35) and (3.36) to (3.32) with the conditions (i) and (ii), we get

$$
\limsup _{n \rightarrow+\infty}\left(\left\|y_{n+1}-y_{n}\right\|-\left\|x_{n+1}-x_{n}\right\|\right) \leq 0
$$

Thus by Lemma 3.4, we conclude that $\lim _{n \rightarrow+\infty}\left\|y_{n}-x_{n}\right\|=0$, which implies that

$$
\lim _{n \rightarrow+\infty}\left\|x_{n+1}-x_{n}\right\|=0
$$

Since $T_{r_{n}}^{F_{1}} x^{*}=x^{*}$ and $T_{r_{n}}^{F_{1}}$ is firmly nonexpansive, we get

$$
\begin{aligned}
\left\|u_{n}-x^{*}\right\|^{2}= & \left\|T_{r_{n}}^{F_{1}}\left(x_{n}+\gamma A^{*}\left(T_{r_{n}}^{F_{2}}-I\right) A x_{n}\right)-x^{*}\right\|^{2} \\
= & \left\|T_{r_{n}}^{F_{1}}\left(x_{n}+\gamma A^{*}\left(T_{r_{n}}^{F_{2}}-I\right) A x_{n}\right)-T_{r_{n}}^{F_{1}} x^{*}\right\|^{2} \\
\leq & \left\langle u_{n}-x^{*}, x_{n}+\gamma A^{*}\left(T_{r_{n}}^{F_{2}}-I\right) A x_{n}-x^{*}\right\rangle \\
= & \frac{1}{2}\left\{\left\|u_{n}-x^{*}\right\|^{2}+\left\|x_{n}+\gamma A^{*}\left(T_{r_{n}}^{F_{2}}-I\right) A x_{n}-x^{*}\right\|^{2}\right. \\
& \left.-\left\|\left(u_{n}-x^{*}\right)-\left[x_{n}+\gamma A^{*}\left(T_{r_{n}}^{F_{2}}-I\right) A x_{n}-x^{*}\right]\right\|^{2}\right\} \\
= & \frac{1}{2}\left\{\left\|u_{n}-x^{*}\right\|^{2}+\left\|x_{n}-x^{*}\right\|^{2}+\gamma(L \gamma-1)\left\|A^{*}\left(T_{r_{n}}^{F_{2}}-I\right) A x_{n}\right\|^{2}\right. \\
& \left.-\left\|u_{n}-x_{n}-\gamma A^{*}\left(T_{r_{n}}^{F_{2}}-I\right) A x_{n}\right\|^{2}\right\} \\
= & \frac{1}{2}\left\{\left\|u_{n}-x^{*}\right\|^{2}+\left\|x_{n}-x^{*}\right\|^{2}-\left[\left\|u_{n}-x_{n}\right\|^{2}\right.\right. \\
& \left.\left.+\gamma^{2}\left\|A^{*}\left(T_{r_{n}}^{F_{2}}-I\right) A x_{n}\right\|^{2}-2 \gamma\left(u_{n}-x_{n}, A^{*}\left(T_{r_{n}}^{F_{2}}-I\right) A x_{n}\right\rangle\right]\right\} \\
= & \frac{1}{2}\left\{\left\|u_{n}-x^{*}\right\|^{2}+\left\|x_{n}-x^{*}\right\|^{2}-\left\|u_{n}-x_{n}\right\|^{2}\right. \\
& \left.+2 \gamma\left\|A\left(u_{n}-x_{n}\right)\right\|\left\|\left(T_{r_{n}}^{F_{2}}-I\right) A x_{n}\right\|\right\} .
\end{aligned}
$$

Hence, we obtain

$$
\left\|u_{n}-x^{*}\right\|^{2} \leq\left\|x_{n}-x^{*}\right\|^{2}-\left\|u_{n}-x_{n}\right\|^{2}+2 \gamma\left\|A\left(u_{n}-x_{n}\right)\right\|\left\|\left(T_{r_{n}}^{F_{2}}-I\right) A x_{n}\right\| .
$$

Again,

$$
\begin{aligned}
& \left\|x_{n+1}-x^{*}\right\|^{2} \\
& =\left\|\left(1-\alpha_{n}\right)\left(x_{n}-x^{*}\right)+\alpha_{n}\left[\tau_{n}\left(S x_{n}-x^{*}\right)+\left(1-\tau_{n}\right)\left(T_{2}^{n} T_{1}^{n} u_{n}-x^{*}\right)\right]\right\|^{2} \\
& =\left\|\left(1-\alpha_{n}\right)\left(x_{n}-x^{*}\right)+\alpha_{n}\left(T_{2}^{n} T_{1}^{n} u_{n}-x^{*}\right)+\alpha_{n} \tau_{n}\left(S x_{n}-T_{2}^{n} T_{1}^{n} u_{n}\right)\right\|^{2} \\
& \leq \quad\left\|\left(1-\alpha_{n}\right)\left(x_{n}-x^{*}\right)+\alpha_{n}\left(T_{2}^{n} T_{1}^{n} u_{n}-x^{*}\right)\right\|^{2} \\
& \quad+2 \tau_{n}\left(S x_{n}-T_{2}^{n} T_{1}^{n} u_{n}, x_{n+1}-x^{*}\right\rangle \\
& \leq \\
& \quad\left(1-\alpha_{n}\right)\left\|x_{n}-x^{*}\right\|^{2}+\alpha_{n}\left\|T_{2}^{n} T_{1}^{n} u_{n}-x^{*}\right\|^{2} \\
& \quad+2 \tau_{n}\left\|S x_{n}-T_{2}^{n} T_{1}^{n} u_{n}\right\|\left\|x_{n+1}-x^{*}\right\|
\end{aligned}
$$




$$
\begin{aligned}
\leq & \left(1-\alpha_{n}\right)\left\|x_{n}-x^{*}\right\|^{2}+\alpha_{n}\left\|u_{n}-x^{*}\right\|^{2} \\
& +2 \tau_{n}\left\|S x_{n}-T_{2}^{n} T_{1}^{n} u_{n}\right\|\left\|x_{n+1}-x^{*}\right\| .
\end{aligned}
$$

Hence, we have

$$
\begin{aligned}
\left\|x_{n+1}-x^{*}\right\|^{2} \leq & \left(1-\alpha_{n}\right)\left\|x_{n}-x^{*}\right\|^{2} \\
& +\alpha_{n}\left(\left\|x_{n}-x^{*}\right\|^{2}+\gamma(L \gamma-1)\left\|\left(T_{r_{n}}^{F_{2}}-I\right) A x_{n}\right\|^{2}\right) \\
& +2 \tau_{n}\left\|S x_{n}-T_{2}^{n} T_{1}^{n} u_{n}\right\|\left\|x_{n+1}-x^{*}\right\|,
\end{aligned}
$$

which gives

$$
\begin{aligned}
\alpha_{n}(1-L \gamma)\left\|\left(T_{r_{n}}^{F_{2}}-I\right) A x_{n}\right\|^{2} \leq & \left\|x_{n+1}-x_{n}\right\|\left(\left\|x_{n}-p\right\|+\left\|x_{n+1}-x^{*}\right\|\right) \\
& +2 \tau_{n}\left\|S x_{n}-T_{2}^{n} T_{1}^{n} u_{n}\right\|\left\|x_{n+1}-x^{*}\right\| .
\end{aligned}
$$

Using the condition (iii) in (3.40), we get

$$
\lim _{n \rightarrow \infty}\left\|\left(T_{r_{n}}^{F_{2}}-I\right) A x_{n}\right\|=0 .
$$

Again,

$$
\begin{aligned}
\left\|x_{n+1}-x^{*}\right\|^{2} \leq & \left(1-\alpha_{n}\right)\left\|x_{n}-x^{*}\right\|^{2}+\alpha_{n}\left\|u_{n}-x^{*}\right\|^{2} \\
& +2 \tau_{n}\left\|S x_{n}-T_{2}^{n} T_{1}^{n} u_{n}\right\|\left\|x_{n+1}-x^{*}\right\| .
\end{aligned}
$$

So, using (3.37) we get

$$
\begin{aligned}
\left\|x_{n+1}-x^{*}\right\|^{2} \leq & \left(1-\alpha_{n}\right)\left\|x_{n}-x^{*}\right\|^{2}+\alpha_{n}\left(\left\|x_{n}-x^{*}\right\|^{2}-\left\|u_{n}-x_{n}\right\|^{2}\right. \\
& \left.+2 \gamma\left\|A\left(u_{n}-x_{n}\right)\right\|\left\|\left(T_{r_{n}}^{F_{2}}-I\right) A x_{n}\right\|\right) \\
& +2 \tau_{n}\left\|S x_{n}-T_{2}^{n} T_{1}^{n} u_{n}\right\|\left\|x_{n+1}-x^{*}\right\|,
\end{aligned}
$$

which gives

$$
\begin{aligned}
\alpha_{n}\left\|u_{n}-x_{n}\right\|^{2} \leq & \left\|x_{n+1}-x_{n}\right\|\left(\left\|x_{n}-x^{*}\right\|+\left\|x_{n+1}-x^{*}\right\|\right) \\
& +2 \tau_{n}\left\|S x_{n}-T_{2}^{n} T_{1}^{n} u_{n}\right\|\left\|x_{n+1}-x^{*}\right\| \\
& +2 \gamma\left\|A\left(u_{n}-x_{n}\right)\right\|\left\|\left(T_{r_{n}}^{F_{2}}-I\right) A x_{n}\right\| .
\end{aligned}
$$

Using the condition (iii) to (3.44), we get

$$
\lim _{n \rightarrow \infty}\left\|u_{n}-x_{n}\right\|=0 .
$$

Using (3.45) we get

$$
\left\|x_{n+1}-u_{n}\right\| \leq\left\|x_{n+1}-x_{n}\right\|+\left\|x_{n}-u_{n}\right\| \rightarrow 0 \quad \text { as } n \rightarrow \infty .
$$


Now

$$
\begin{aligned}
\left\|T_{2}^{n} T_{1}^{n} u_{n}-u_{n}\right\| \leq & \left\|T_{2}^{n} T_{1}^{n} u_{n}-y_{n}\right\|+\left\|y_{n}-u_{n}\right\| \\
\leq & \tau_{n}\left\|T_{2}^{n} T_{1}^{n} u_{n}-S x_{n}\right\|+\left\|y_{n}-u_{n}\right\| \\
\leq & \tau_{n}\left\|x_{n}-S x_{n}\right\|+\tau_{n}\left\|T_{2}^{n} T_{1}^{n} u_{n}-x_{n}\right\|+\left\|y_{n}-u_{n}\right\| \\
\leq & \tau_{n}\left\|x_{n}-S x_{n}\right\|+\tau_{n}\left\|T_{2}^{n} T_{1}^{n} u_{n}-u_{n}\right\|+\tau_{n}\left\|u_{n}-x_{n}\right\| \\
& +\left\|y_{n}-u_{n}\right\| .
\end{aligned}
$$

Therefore, we have

$$
\begin{aligned}
\left\|T_{2}^{n} T_{1}^{n} u_{n}-u_{n}\right\| \leq & \frac{\tau_{n}}{1-\tau_{n}}\left\|x_{n}-S x_{n}\right\|+\frac{\tau_{n}}{1-\tau_{n}}\left\|u_{n}-x_{n}\right\| \\
& +\frac{1}{1-\tau_{n}}\left\|y_{n}-u_{n}\right\| .
\end{aligned}
$$

Since $\alpha_{n}\left\|x_{n}-y_{n}\right\|=\left\|x_{n+1}-x_{n}\right\|,\left\|x_{n}-y_{n}\right\| \rightarrow 0$ as $n \rightarrow \infty$. So,

$$
\left\|y_{n}-u_{n}\right\| \leq\left\|y_{n}-x_{n}\right\|+\left\|x_{n}-u_{n}\right\| \rightarrow 0 \quad \text { as } n \rightarrow \infty
$$

Hence,

$$
\left\|T_{2}^{n} T_{1}^{n} u_{n}-u_{n}\right\| \rightarrow 0 \quad \text { as } n \rightarrow \infty
$$

Now, we show that

$$
\limsup _{n \rightarrow \infty}\left\langle S p-p, x_{n}-p\right\rangle \leq 0
$$

where $p$ is the unique solution of the variational inequality (3.24). Since $\left\{x_{n}\right\}$ is bounded, there exists a subsequence $\left\{x_{n_{j}}\right\}$ of $\left\{x_{n}\right\}$ such that $x_{n_{j}} \rightarrow \bar{x}$ as $j \rightarrow \infty$ and

$$
\limsup _{n \rightarrow \infty}\left\langle S p-p, x_{n}-p\right\rangle=\lim _{j \rightarrow \infty}\left\langle S p-p, x_{n_{j}}-p\right\rangle
$$

Since $\left\|x_{n}-u_{n}\right\| \rightarrow 0$ as $n \rightarrow \infty, u_{n_{j}} \rightarrow \bar{x}$. Now, following similar steps to Theorem 3.1, we can show that $\bar{x} \in \Gamma \cap \mathcal{S}$. Hence

$$
\lim _{j \rightarrow \infty}\left\langle S p-p, x_{n_{j}}-p\right\rangle=\langle S p-p, \bar{x}-p\rangle \leq 0
$$

Finally, we show that $x_{n} \rightarrow p$ and $n \rightarrow \infty$. From (2.1) and (3.23), we have

$$
\begin{aligned}
\left\|x_{n+1}-p\right\|^{2}= & \left\|\left(1-\alpha_{n}\right)\left(x_{n}-p\right)+\alpha_{n}\left[\tau_{n} S x_{n}+\left(1-\tau_{n}\right) T_{2}^{n} T_{1}^{n} u_{n}-p\right]\right\|^{2} \\
= & \left\|\left(1-\alpha_{n}\right)\left(x_{n}-p\right)+\alpha_{n}\left(1-\tau_{n}\right)\left(T_{2}^{n} T_{1}^{n} u_{n}-p\right)+\alpha_{n} \tau_{n}\left(S x_{n}-p\right)\right\|^{2} \\
\leq & \left\|\left(1-\alpha_{n}\right)\left(x_{n}-p\right)+\alpha_{n}\left(1-\tau_{n}\right)\left(T_{2}^{n} T_{1}^{n} u_{n}-p\right)\right\|^{2} \\
& +2 \alpha_{n} \tau_{n}\left\langle S x_{n}-p, x_{n+1}-p\right\rangle
\end{aligned}
$$




$$
\begin{aligned}
\leq & \left(1-\alpha_{n}\right)\left\|x_{n}-p\right\|^{2}+\alpha_{n}\left(1-\tau_{n}\right)^{2}\left\|T_{2}^{n} T_{1}^{n} u_{n}-p\right\|^{2} \\
& +2 \alpha_{n} \tau_{n}\left\langle S x_{n}-p, x_{n+1}-p\right\rangle \\
\leq & \left(1-\alpha_{n}+\alpha_{n}\left(1-\tau_{n}\right)^{2}\right)\left\|x_{n}-p\right\|^{2}+2 \alpha_{n} \tau_{n}\left\langle S x_{n}-S p, x_{n+1}-p\right\rangle \\
& +2 \alpha_{n} \tau_{n}\left\langle S p-p, x_{n+1}-p\right\rangle \\
\leq & \left(1-\alpha_{n}+\alpha_{n}\left(1-\tau_{n}\right)^{2}\right)\left\|x_{n}-p\right\|^{2}+2 \alpha_{n} \tau_{n}\left\|S x_{n}-S p\right\|\left\|x_{n+1}-p\right\| \\
& +2 \alpha_{n} \tau_{n}\left\langle S p-p, x_{n+1}-p\right\rangle \\
\leq & \left(1-\alpha_{n}+\alpha_{n}\left(1-\tau_{n}\right)^{2}\right)\left\|x_{n}-p\right\|^{2} \\
& +\alpha_{n} \tau_{n}\left[\left\|S x_{n}-S p\right\|^{2}+\left\|x_{n+1}-p\right\|^{2}\right]+2 \alpha_{n} \tau_{n}\left\langle S p-p, x_{n+1}-p\right\rangle \\
\leq & \left(1-\alpha_{n}+\alpha_{n}\left(1-\tau_{n}\right)^{2}+\alpha_{n} \tau_{n} \rho\right)\left\|x_{n}-p\right\|^{2}+\alpha_{n} \tau_{n}\left\|x_{n+1}-p\right\|^{2} \\
& +2 \alpha_{n} \tau_{n}\left\langle S p-p, x_{n+1}-p\right\rangle \\
= & \left(1-\alpha_{n} \tau_{n}-\alpha_{n} \tau_{n}\left(1-\rho-\tau_{n}\right)\right)\left\|x_{n}-p\right\|^{2}+\alpha_{n} \tau_{n}\left\|x_{n+1}-p\right\|^{2} \\
& +2 \alpha_{n} \tau_{n}\left\langle S p-p, x_{n+1}-p\right\rangle .
\end{aligned}
$$

From the above inequality, it follows that

$$
\begin{aligned}
\left\|x_{n+1}-p\right\|^{2} \leq & \left(1-\frac{\alpha_{n} \tau_{n}\left(1-\rho-\tau_{n}\right)}{1-\alpha_{n} \tau_{n}}\right)\left\|x_{n}-p\right\|^{2} \\
& +2 \frac{\alpha_{n} \tau_{n}}{1-\alpha_{n} \tau_{n}}\left\langle S p-p, x_{n+1}-p\right\rangle .
\end{aligned}
$$

Considering $a_{n}=\left\|x_{n}-p\right\|^{2}, b_{n}=\frac{\alpha_{n} \tau_{n}\left(1-\rho-\tau_{n}\right)}{1-\alpha_{n} \tau_{n}}$ and $c_{n}=2 \frac{\alpha_{n} \tau_{n}}{1-\alpha_{n} \tau_{n}}\left\langle S p-p, x_{n+1}-p\right\rangle$, we get $a_{n+1} \leq$ $\left(1-b_{n}\right) a_{n}+c_{n}$. Hence, from Lemma 3.5, we conclude that $\left\{x_{n}\right\}$ converges strongly to $p$.

The following consequence is a strong convergence theorem for computing a common solution of an equilibrium problem and a hierarchical fixed point problem in a real Hilbert space.

Corollary 3.7 Let $H_{1}$ be a Hilbert spaces. Let $C$ be nonempty closed and convex subset of $H_{1}$. Let $F_{1}: C \times C \rightarrow \mathbb{R}$ and be a bifunction satisfying Assumption A. Let $S: C \rightarrow C$ be a contraction mapping with coefficient $\rho \geq 0$ and $\left\{T_{i}\right\}_{i=1}^{N}: C \rightarrow H_{1}$ be $k_{i}$-strictly pseudocontractive nonself-mappings. Assume that $E P(F, C) \cap \mathcal{S} \neq \emptyset$. Define a sequence $\left\{x_{n}\right\}$ as follows:

$$
\left\{\begin{array}{l}
x_{0} \in C \\
u_{n}=T_{r_{n}}^{F_{1}}\left(x_{n}\right), \quad n \geq 1 \\
x_{n+1}=\left(1-\alpha_{n}\right) x_{n}+\alpha_{n}\left(\tau_{n} S x_{n}+\left(1-\tau_{n}\right) T_{N}^{n} T_{N-1}^{n} \cdots T_{1}^{n} u_{n}\right)
\end{array}\right.
$$

where $T_{i}^{n}=\left(1-\delta_{n}^{i}\right) I+\delta_{n}^{i} P_{C}\left(\beta_{i} I+\left(1-\beta_{i}\right) T_{i}\right), 0 \leq k_{i} \leq \beta_{i}<1$, and $\delta_{n}^{i} \in(0,1)$ for $i=1,2, \ldots, N$. Let $\left\{\alpha_{n}\right\}$ and $\left\{\tau_{n}\right\}$ be two real sequences in $(0,1)$. Suppose the following conditions (i)-(iv) of Theorem 3.6 are satisfied. Then the sequence $\left\{x_{n}\right\}$ converges strongly to $p \in \operatorname{EP}(F, C) \cap \mathcal{S}$, which is the unique solution of the following variational inequality:

$$
\langle p-S p, p-y\rangle \leq 0, \quad \forall y \in E P(F, C) \cap \mathcal{S} .
$$


Proof Taking $A=0$ in Theorem 3.6, the conclusion of Corollary 3.7 is followed.

\section{Conclusions}

In this paper, we have introduced a modified Krasnoselski-Mann type iterative method for approximating a common solution of a split mixed equilibrium problem and a hierarchical fixed point problem of a finite collection of $k$-strictly pseudocontractive nonselfmappings.

Our main results improve and extend the corresponding results of Moudafi and Mainge [24], Moudafi [22] and Kazmi et al. [13] from single nonexpansive self-mapping to a finite collection of $k_{i}$-strictly pseudocontractive nonself-mappings. Also, we have studied our iterative algorithm by giving an explicit formula for selecting the step size so that the implementation of the proposed algorithm does not require any prior information of operator norm. We also have established strong convergence results for a special class of hierarchical fixed point and split mixed equilibrium problem.

In [5], Ceng and Petruşel have introduced a cyclic algorithm for HFPP of a finite collection of nonexpansive nonself-mappings in Banach spaces. Whether we can extend Theorems 3.1 and 3.6 to Banach spaces will be an issue of future research.

Funding

This work was supported by the Basic Science Research Program through the National Research Foundation (NRF) Grant funded by Ministry of Education of the republic of Korea (2018R1D1A1B07045427).

Availability of data and materials

Not applicable.

Competing interests

The authors declare that they have no competing interests.

Authors' contributions

The authors contribute equally. Both authors read and approved the final version of the manuscript.

\section{Author details}

'Department of Mathematics Education, Kyungnam University, Changwon, Gyeongnam, 51767, Korea. ${ }^{2}$ Department of Mathematics, Motilal Nehru National Institute of Technology Allahabad, Prayagraj, 211004, India.

\section{Publisher's Note}

Springer Nature remains neutral with regard to jurisdictional claims in published maps and institutional affiliations.

Received: 12 May 2020 Accepted: 17 September 2020 Published online: 29 September 2020

\section{References}

1. Blum, E., Oettli, W.: From optimization and variational inequalities to equilibrium problems. Math. Stud. 63(1), 123-145 (1994)

2. Brezis, H.: Operateurs maximaux monotones et semi-groupes de contractions dans lesespaces de Hilbert, vol. 5. Elsevier, Amsterdam (1973)

3. Buong, N., Duong, L.T.: An explicit iterative algorithm for a class of variational inequalities in Hilbert spaces. J. Optim. Theory Appl. 151(3), 513-524 (2011)

4. Byrne, C.: A unified treatment of some iterative algorithms in signal processing and image reconstruction. Inverse Probl. 20(1), 103-120 (2004)

5. Ceng, L.C., Petrusel, A.: Krasnoselski-Mann iterations for hierarchical fixed point problems for a finite family of nonself mappings in Banach spaces. J. Optim. Theory Appl. 146(3), 617-639 (2010)

6. Ceng, L.C., Petrusel, A., Yao, J.C., Yao, Y.: Systems of variational inequalities with hierarchical variational inequality constraints for Lipschitzian pseudocontractions. Fixed Point Theory 20, 113-133 (2019)

7. Censor, Y., Gibali, A., Reich, S.: Algorithms for the split variational inequality problem. Numer. Algorithms 59(2), 301-323 (2012)

8. Combettes, P.L.: Quasi-Fejerian analysis of some optimization algorithms. In: Butnariu, D., Censor, Y., Reich, S. (eds.) Inherently Parallel Algorithms in Feasibility and Optimization and Their Applications, pp. 115-152. Elsevier, New York (2001)

9. Combettes, P.L., Hirstoaga, S.A.: Equilibrium programming in Hilbert spaces. J. Nonlinear Convex Anal. 6(1), 117-136 (2005) 
10. Deepho, J., Kumam, W., Kumam, P.: A new hybrid projection algorithm for solving the split generalized equilibrium problems and the system of variational inequality problems. J. Math. Model. Algorithms Oper. Res. 13(4), 405-423 (2014)

11. Farid, M.: The subgradient extragradient method for solving mixed equilibrium problems and fixed point problems in Hilbert spaces. J. Appl. Numer. Optim. 1, 335-345 (2019)

12. Goebel, K., Kirk, W.A.: Topics in Metric Fixed Point Theory. Cambridge Studies in Advanced Math., vol. 28. Cambridge University Press, Cambridge (1990)

13. Kazmi, K., Ali, R., Furkan, M.: Krasnoselski-Mann type iterative method for hierarchical fixed point problem and split mixed equilibrium problem. Numer. Algorithms 74(1), 1-20 (2017)

14. Kim, J.K.: Strong convergence theorems by hybrid projection methods for equilibrium problems and fixed point problems of the asymptotically quasi-nonexpansive mappings. Fixed Point Theory Appl. (2011). https://doi.org/10.1186/1687-1812-2011-10

15. Kim, J.K.: Convergence theorems of iterative sequences for generalized equilibrium problems involving strictly pseudocontractive mappings in Hilbert spaces. J. Comput. Anal. Appl. 18(3), 454-471 (2015)

16. Kim, J.K., Lim, W.H.: A new iterative algorithm of pseudomonotone mappings for equilibrium problems in Hilbert spaces. J. Inequal. Appl. 2013, 128 (2013)

17. Kim, J.K., Tuyen, T.M.: On the some regularization methods for common fixed point of a finite family of nonexpansive mappings. J. Nonlinear Convex Anal. 17(1), 99-104 (2016)

18. Majee, P., Nahak, C.: A hybrid viscosity iterative method with averaged mappings for split equilibrium problems and fixed point problems. Numer. Algorithms 74(2), 609-635 (2017)

19. Majee, P., Nahak, C.: Inertial algorithms for a system of equilibrium problems and fixed point problems. Rend. Circ. Mat. Palermo 2(68), 11-27 (2018)

20. Majee, P., Nahak, C.: A modified iterative method for capturing a common solution of split generalized equilibrium problem and fixed point problem. Rev. R. Acad. Cienc. Exactas Fís. Nat., Ser. A Mat. 112(4), 1327-1348 (2018)

21. Marino, G., Colao, V., Muglia, L., Yao, Y.: Krasnoselski-Mann iteration for hierarchical fixed points and equilibrium problem. Bull. Aust. Math. Soc. 79(2), 187-200 (2009)

22. Moudafi, A.: Krasnoselski-Mann iteration for hierarchical fixed point problems. Inverse Probl. 23(4), 16-35 (2007)

23. Moudafi, A.: Split monotone variational inclusions. J. Optim. Theory Appl. 150(2), 275-283 (2011)

24. Moudafi, A., Mainge, P.E.: Towards viscosity approximations of hierarchical fixed point problems. Fixed Point Theory Appl. 2006, $95453(2006)$

25. Shahzad, N., Zegeye, H.: Convergence theorems of common solutions for fixed point, variational inequality and equilibrium problems. J. Nonlinear Var. Anal. 3, 189-203 (2019)

26. Suzuki, T.: Strong convergence of Krasnoselski and Mann type sequences for one parameter nonexpansive semigroups without Bochner integrals. J. Math. Anal. Appl. 305(1), 227-239 (2005)

27. Takahahsi, W., Yao, J.C.: The split common fixed point problem for two finite families of nonlinear mappings in Hilbert spaces. J. Nonlinear Convex Anal. 20, 173-195 (2019)

28. Wang, S., Liu, X., An, Y.S.: A new iterative algorithm for generalized split equilibrium problem in Hilbert spaces. Nonlinear Funct. Anal. Appl. 22(4), 911-924 (2017)

29. Wang, S., Zhang, Y., Wang, W.: Extragradient algorithms for split pseudomonotone equilibrium problems and fixed point problems in Hilbert spaces. J. Nonlinear Funct. Anal. 2019, Article ID 26 (2019)

30. Wang, Z.M.: Convergence theorems based on the shrinking projection method for hemi-relatively nonexpansive mappings, variational inequalities and equilibrium problem. Nonlinear Funct. Anal. Appl. 22(3), 459-483 (2017)

31. Xu, H.K.: Iterative algorithms for nonlinear operator. J. Lond. Math. Soc. 66, 240-256 (2002)

32. Xu, H.K.: A variable Krasnosel'skii-Mann algorithm and the multiple-set split feasibility problem. Inverse Probl. 22(6), 2021-2034 (2006)

33. Yamada, I., Ogura, N., Shirakawa, N.: A numerically robust hybrid steepest descent method for the convexly constrained generalized inverse problems. Contemp. Math. 313, 269-305 (2002)

34. Yang, Q., Zhao, J.: Generalized KM theorems and their applications. Inverse Probl. 22(3), $833-844$ (2006)

35. Yao, Y., Liou, Y.C.: Weak and strong convergence of Krasnoselski-Mann iteration for hierarchical fixed point problems. Inverse Probl. 24(1), 015015 (2008)

36. Yao, Y., Shahzad, N., Yao, J.C.: Projected subgradient algorithms for pseudomonotone equilibrium problems and fixed points of pseudocontractive operators. Mathematics 8, Article ID 461 (2020)

37. Zhou, H.: Convergence theorems of fixed points for $k$-strict pseudo-contractions in Hilbert spaces. Nonlinear Anal. TMA 69(2), 456-462 (2008)

\section{Submit your manuscript to a SpringerOpen ${ }^{\circ}$ journal and benefit from:}

- Convenient online submission

- Rigorous peer review

- Open access: articles freely available online

- High visibility within the field

Retaining the copyright to your article

Submit your next manuscript at $\boldsymbol{~ s p r i n g e r o p e n . c o m ~}$ 